\title{
THE INVERSE APPROACH TO DIRAC-TYPE SYSTEMS BASED ON THE $A$-FUNCTION CONCEPT
}

\author{
FRITZ GESZTESY AND ALEXANDER SAKHNOVICH
}

ABstract. The principal objective in this paper is a new inverse approach
general Dirac-type systems of the form
\[ y^{\prime}(x, z)=i(z J+J V(x)) y(x, z) \quad(x \geq 0) \]
where $y=\left(y_{1}, \ldots, y_{m}\right)^{\top}$ and (for $\left.m_{1}, m_{2} \in \mathbb{N}\right)$
\[ J=\left[\begin{array}{cc}I_{m_{1}} & 0_{m_{1} \times m_{2}} \\ 0_{m_{2} \times m_{1}} & -I_{m_{2}}\end{array}\right], \quad V=\left[\begin{array}{cc}0_{m_{1}} & v \\ v^{*} & 0_{m_{2}}\end{array}\right], \quad m_{1}+m_{2}=: m, \]

for $v \in\left[C^{1}([0, \infty))\right]^{m_{1} \times m_{2}}$, modeled after B. Simon's 1999 inverse approach to half-line Schrödinger operators. In particular, we derive the $\mathcal{A}$-equation associated to this Dirac-type system in the ( $z$-independent) form

$$
\frac{\partial}{\partial \ell} \mathcal{A}(x, \ell)=\frac{\partial}{\partial x} \mathcal{A}(x, \ell)+\int_{0}^{x} \mathcal{A}(x-t, \ell) \mathcal{A}(0, \ell)^{*} \mathcal{A}(t, \ell) d t \quad(x \geq 0, \ell \geq 0) .
$$

Given the fundamental positivity condition $S_{T}>0$ in 1.14) (cf. 1.13) for details), we prove that this integro-differential equation for $\mathcal{A}(\cdot, \cdot)$ is uniquely solvable for initial conditions

$$
\mathcal{A}(\cdot, 0)=\mathcal{A}(\cdot) \in\left[C^{1}([0, \infty))\right]^{m_{2} \times m_{1}}
$$

and the corresponding potential coefficient $v \in\left[C^{1}([0, \infty))\right]^{m_{1} \times m_{2}}$ can be recovered from $\mathcal{A}(\cdot, \cdot)$ via

$$
v(\ell)=-i \mathcal{A}(0, \ell)^{*} \quad(\ell \geq 0) .
$$

Contents

1. Introduction

2. Preliminaries

3. The $\mathcal{A}$-Function for Dirac-Type Systems: Part I

4. The $\mathcal{A}$-Function for Dirac-Type Systems: Part II

5. The $\mathcal{A}$-Equation for Dirac-Type Systems

6. The Inverse Approach

Appendix A. Various Results in Support of Step 2

References in the Proof of Theorem 5.1

\section{INTRODUCTION}

Barry Simon's seminal paper [31] on a new approach to inverse spectral problems for scalar Schrödinger operators $\left(-d^{2} / d x^{2}\right)+V(x)$ on the half-line $(0, \infty)$ with a

Date: March 5, 2019.

2010 Mathematics Subject Classification. Primary 34A55, 34B20, 34L40; Secondary 34B24.

Key words and phrases. Inverse problems, Dirac-type systems, matrix-valued potentials.

The research of A. L. Sakhnovich was supported by the Austrian Science Fund (FWF) under Grant No. P29177. 
Dirichlet boundary condition at $x=0$ (for simplicity), is based on the so-called $A$-equation

$$
\frac{\partial A}{\partial x}(\alpha, x)=\frac{\partial A}{\partial \alpha}(\alpha, x)+\int_{0}^{\alpha} A(\beta, x) A(\alpha-\beta, x) d \beta \quad(x \geq 0, \alpha \geq 0) .
$$

The $A$-function $A(\alpha, x)$ on the other hand is connected with the Weyl-Titchmarsh $m$-function $m(z, x)$ of the Schrödinger equation on the half-line $[x, \infty)(x>0)$ via the Laplace (or Fourier) transformation in the complex domain. More precisely, assuming $V \in L^{1}((0, T))$ for all $T \in(0, \infty)$ to be real-valued, one has

$$
\begin{array}{r}
m(z, x)=i z^{1 / 2}-\int_{0}^{T} A(\alpha, x) e^{2 i \alpha z^{1 / 2}} d \alpha+O\left(e^{i(2 T-\varepsilon) z^{1 / 2}}\right), \\
z \in \mathbb{C} \backslash[0, \infty), \pi-\varepsilon<\arg \left(i z^{1 / 2}\right)<(\pi / 2)+\varepsilon,
\end{array}
$$

for any $T>\varepsilon>0$. In particular, one notes that (1.1) is independent of the spectral parameter $z$.

Introducing the set

$$
\boldsymbol{A}_{T}=\left\{A \in L^{1}([0, T]) \mid A \text { real-valued, } I+\mathcal{K}_{A}>0\right\},
$$

where

$$
\begin{aligned}
& \left(\mathcal{K}_{A} f\right)(\alpha)=\int_{0}^{T} K(\alpha, \beta) f(\beta) d \beta, \quad \alpha \in[0, T], f \in L^{2}((0, T)), \\
& K(\alpha, \beta)=[\phi(\alpha-\beta)-\phi(\alpha+\beta)] / 2, \quad \phi(\alpha)=\int_{0}^{|\alpha| / 2} A(\gamma) d \gamma, \quad(\alpha, \beta \in[0, T]),
\end{aligned}
$$

Remling 24] completed the inverse approach based on the $A$-function by proving that $\boldsymbol{A}_{T}$ is precisely the set of $A$-functions in

$$
\begin{array}{r}
m(z)=i z^{1 / 2}-\int_{0}^{T} A(\alpha) e^{2 i \alpha z^{1 / 2}} d \alpha+O\left(e^{i(2 T-\varepsilon) z^{1 / 2}}\right), \\
z \in \mathbb{C} \backslash[0, \infty), \pi-\varepsilon<\arg \left(i z^{1 / 2}\right)<(\pi / 2)+\varepsilon,
\end{array}
$$

for all $T>0$. Equivalently, given $A_{0} \in L^{1}((0, T))$, there exists a potential $V \in$ $L^{1}((0, T))$ such that $A_{0}$ is the $A$-function of $V$ if and only if $A_{0} \in \boldsymbol{A}_{T}$. In particular, we emphasize that the $A$-equation (1.1) plays a crucial role in Simon's new inverse approach to half-line Schrödinger operators (in contrast to the traditional approach in [3, [17]- 19], 21, 23]), since

$$
V(x)=A(0, x) \text { for a.e. } x \in[0, T] .
$$

Additional work on the $A$-function ensued in [14, and other interesting results by different authors followed (see, e.g., [16, 24, 32, 33], and the survey article [9]).

Although Dirac-type systems present a natural generalization of the Schrödinger equation and there are many analogies between the corresponding spectral and inverse spectral theories, the analog of the $A$-equation for Dirac-type system (which will be called the $\mathcal{A}$-equation to distinguish it from the Schrödinger equation case) was not formulated until now. In the present paper we fill this gap and formulate and also prove solvability of the $\mathcal{A}$-equation for the general Dirac-type system (in the general matrix-valued case)

$$
y^{\prime}(x, z)=i(z J+J V(x)) y(x, z), \quad x \geq 0 \quad\left(y^{\prime}(x, z):=\frac{d y}{d x}(x, z)\right),
$$


where $y=\left(y_{1}, \ldots, y_{m}\right)^{\top}$ and $\left(\right.$ for $\left.m_{1}, m_{2} \in \mathbb{N}\right)$

$$
J=\left[\begin{array}{cc}
I_{m_{1}} & 0_{m_{1} \times m_{2}} \\
0_{m_{2} \times m_{1}} & -I_{m_{2}}
\end{array}\right], \quad V=\left[\begin{array}{cc}
0_{m_{1}} & v \\
v^{*} & 0_{m_{2}}
\end{array}\right], \quad m_{1}+m_{2}=: m .
$$

Here $\mathbb{N}$ stands for the set of natural numbers and $I_{k}$ is the $k \times k$ identity matrix. The $m \times m$ matrix-valued function $V$ (and, sometimes, the $m_{1} \times m_{2}$ matrix-valued function $v$ ) is called the potential of the Dirac-type system (1.6).

Before being able to formulate some of our principal results, we need a few preparations and some standard definitions.

Notation 1.1. The $m \times m$ fundamental solution of system (1.6), normalized by $I_{m}$ at $x=\ell$, is denoted by $u_{\ell}(x, z)$, that is, one has,

$$
u_{\ell}(\ell, z)=I_{m} \text {. }
$$

Definition 1.2. Suppose that $v \in\left[L^{1}((0, R))\right]^{m_{1} \times m_{2}}$ for all $R>0$. Then the $m_{2} \times$ $m_{1}$ matrix function $\varphi_{\ell}(z)$ is called a (matrix-valued) Weyl-Titchmarsh function associated with the system (1.6) on $[\ell, \infty)(\ell \geq 0)$ if it is holomorphic in $\mathbb{C}_{+}$and the entries of $u_{\ell}(\cdot, z)\left[\begin{array}{c}I_{m_{1}} \\ \varphi_{\ell}(z)\end{array}\right]$ belong to $L^{2}((\ell, \infty))$, that is,

$$
u_{\ell}(\cdot, z)\left[\begin{array}{c}
I_{m_{1}} \\
\varphi_{\ell}(z)
\end{array}\right] \in\left[L^{2}((\ell, \infty))\right]^{m \times m_{1}} .
$$

Remark 1.3. The Weyl-Titchmarsh function $\varphi_{\ell}(\cdot, z)$ always exists, is unique, and contractive for $z \in \mathbb{C}_{+}$(see [8] and [28, Prop. 2.17 and Cor. 2.21]).

Propositions 3.5 and 4.1, and Remark 4.2 in the present paper then yield the next statement:

Proposition 1.4. Assume that $v \in\left[C^{1}([0, \infty))\right]^{m_{1} \times m_{2}}$ in the Dirac-type system (1.6). Then, the associated Weyl-Titchmarsh functions $\varphi_{\ell}(z)$ admit a representation of the type

$$
\varphi_{\ell}(z)=2 i z \int_{0}^{\infty} e^{2 i x z} \Phi(x, \ell) d x \quad(\operatorname{Im}(z)>0, \ell \geq 0),
$$

where

$$
\Phi(\cdot, \ell) \in\left[C^{2}([0, \infty))\right]^{m_{2} \times m_{1}} \quad(\ell \geq 0) .
$$

The analog of the $A$-function in (1.2) for the Dirac-type system (1.6) (which will be called the $\mathcal{A}$-function) can now be introduced via

$$
\mathcal{A}(x, \ell):=\frac{\partial}{\partial x} \Phi(x, \ell) \equiv \Phi^{\prime}(x, \ell) ; \quad \mathcal{A}(x):=\mathcal{A}(x, 0) \quad(x \geq 0, \ell \geq 0) .
$$

According to Proposition 1.4 and Corollary 6.1 the following result holds.

Proposition 1.5. Assume that $v \in\left[C^{1}([0, \infty))\right]^{m_{1} \times m_{2}}$ in the Dirac-type system (1.6). Then, with $\mathcal{A}(\cdot)$ given by (1.12), the operator $S:=S_{T}$ in $\left[L^{2}((0, T))\right]^{m_{2}}$ $(T \in(0, \infty))$ introduced as

$$
\begin{aligned}
& (S f)(x)=\left(S_{T} f\right)(x)=f(x)-\int_{0}^{T} s(x, t) f(t) d t, \quad f \in\left[L^{2}((0, T))\right]^{m_{2}}, \\
& s(x, t):=\int_{0}^{\min (x, t)} \mathcal{A}(x-\xi) \mathcal{A}(t-\xi)^{*} d \xi \quad((x, t) \in(0, T) \times(0, T)),
\end{aligned}
$$


satisfies the positive definiteness property

$$
S=S_{T}>0
$$

for all $T \in(0, \infty)$.

Now, we are in a position to formulate our principal theorem, an immediate consequence of Theorems 5.1 and 6.3, and formula (4.33):

Theorem 1.6. Suppose that $v \in\left[C^{1}([0, \infty))\right]^{m_{1} \times m_{2}}$ in the Dirac-type system (1.6). Then, the $\mathcal{A}(\cdot, \cdot)$-function given by (1.12) is continuously differentiable, $\mathcal{A}(\cdot, \cdot) \in\left[C^{1}\left(\mathbb{R}_{+,+}^{2}\right)\right]^{m_{2} \times m_{1}}$, with $\mathbb{R}_{+,+}^{2}=\left\{(x, \ell) \in \mathbb{R}^{2} \mid x \geq 0, \ell \geq 0\right\}$, and satisfies the integro-differential equation $\left(\right.$ for $\left.(x, \ell) \in \mathbb{R}_{+,+}^{2}\right)$

$$
\frac{\partial}{\partial \ell} \mathcal{A}(x, \ell)=\frac{\partial}{\partial x} \mathcal{A}(x, \ell)+\int_{0}^{x} \mathcal{A}(x-t, \ell) \mathcal{A}(0, \ell)^{*} \mathcal{A}(t, \ell) d t .
$$

Conversely, the system (1.15) with initial condition

$$
\mathcal{A}(\cdot, 0)=\mathcal{A}(\cdot) \in\left[C^{1}([0, \infty))\right]^{m_{2} \times m_{1}},
$$

such that the positive definiteness property (1.14) holds for all $T \in(0, \infty)$, has a unique solution

$$
\mathcal{A}(\cdot, \cdot) \in\left[C^{1}\left(\mathbb{R}_{+,+}^{2}\right)\right]^{m_{2} \times m_{1}},
$$

and an associated Dirac-type system of the form (1.6) exists. In particular, the potential coefficient $v \in\left[C^{1}([0, \infty))\right]^{m_{1} \times m_{2}}$ in this Dirac-type system is recovered from $\mathcal{A}(0, \cdot)$ via

$$
v(\ell)=-i \mathcal{A}(0, \ell)^{*} \quad(\ell \geq 0)
$$

One notes that in analogy to (1.1), (1.15) is also independent of the spectral parameter $z$.

Given these facts, we can summarize the direct and inverse $\mathcal{A}$-function problem as follows upon introducing the set

$$
\begin{array}{r}
\mathcal{A}=\left\{\mathcal{A} \in\left[C^{1}([0, \infty))\right]^{m_{2} \times m_{1}} \mid S:=S_{T} \text { defined in (1.13) satisfies } S_{T}>0\right. \\
\text { for all } T \in(0, \infty)\} .
\end{array}
$$

\section{Direct Problem:}

$$
\begin{gathered}
v \in\left[C^{1}([0, \infty))\right]^{m_{1} \times m_{2}} \longrightarrow \varphi_{\ell}(z) \frac{\text { by }(1.10)}{\text { inverse Laplace transform }} \Phi(x, \ell),(x, \ell) \in \mathbb{R}_{+,+}^{2} \\
\stackrel{\text { by } \stackrel{1.122}{\longrightarrow}}{\longrightarrow} \mathcal{A}(x, \ell)=\frac{\partial}{\partial x} \Phi(x, \ell), \quad(x, \ell) \in \mathbb{R}_{+,+}^{2} \longrightarrow \mathcal{A}(\cdot, 0):=\mathcal{A}(\cdot) \in \mathcal{A} .
\end{gathered}
$$

\section{Inverse Problem:}

$$
\begin{gathered}
\mathcal{A}(\cdot) \in \mathcal{A} \stackrel{\text { by }(1.15),(1.16)}{\longrightarrow} \mathcal{A}(\cdot, \cdot) \in\left[C^{1}\left(\mathbb{R}_{+,+}^{2}\right)\right]^{m_{2} \times m_{1}} \\
\stackrel{\text { by }(1.18)}{\longrightarrow} v=-i \mathcal{A}(0, \cdot)^{*} \in\left[C^{1}([0, \infty))\right]^{m_{1} \times m_{2}} .
\end{gathered}
$$

While we focus for convenience on half-line Dirac-type systems (1.6) and global solutions $\mathcal{A}(\cdot, \cdot)$ of $(1.15)$ on $\mathbb{R}_{+,+}^{2}$, our results apply locally just as well; this fact 
is further discussed in Remark 6.4 and in the local Borg-Marchenko uniqueness result, Theorem 6.5.

Although the $\mathcal{A}$-function equation is particularly interesting in connection with inverse problems, we here note that it also characterizes shifts (i.e., translations) of Dirac-type systems. As one of our results that is of independent interest, we also mention the series representation of the Weyl-Titchmarsh function, which was derived in the process of the proof of Theorem 5.1 (see Lemma A.3).

Remark 1.7. (i) Our results apply, in particular, to the special class of $m_{1} \times m_{1}$ matrix-valued Schrödinger operators. The latter case is equivalent to the subclass $m_{1}=m_{2}$ of the so called supersymmetric Dirac-type operators for which $v=-v^{*}$ holds. For a detailed discussion of supersymmetric Dirac-type operators and their close (essentially, one-to-one) relations with (matrix-valued) Schrödinger operators via the celebrated Miura-type transformations (i.e., via appropriate Riccati-type relations) we refer, for instance, to [4, 6, 7, 10, 13.

(ii) In the special scalar context, $m_{1}=m_{2}=1$, the fact (1.21) represents the Diractype analog of the Schrödinger operator results (1.3)-(1.5) due to Remling [24].

The precise connection between the $\mathcal{A}$-function and the underlying matrix-valued spectral function, and hence the connection between $\mathcal{A}$ and inverse spectral theory, is in preparation 12 .

Finally, we briefly summarize the notation used in this paper: $I_{r}$ and $0_{r}$ are the $r \times r$ identity and zero matrix in $\mathbb{C}^{r}$, respectively $(r \in \mathbb{N})$. Similarly, $0_{r_{1} \times r_{2}}$ represents the (rectangular) $r_{1} \times r_{2}$ zero matrix $\left(r_{1}, r_{2} \in \mathbb{N}\right)$.

Throughout this paper, for $X$ a given space, $A \in X^{r_{1} \times r_{2}}$ represents an $r_{1} \times r_{2}$ block (operator) matrix $A$ with entries in $X\left(r_{1}, r_{2} \in \mathbb{N}\right)$. If $r_{2}=1$, we use the notation $A \in X^{r_{1}}$.

We use the abbreviation $L^{p}(\Omega) \equiv L^{p}(\Omega ; d x)(\Omega \subseteq \mathbb{R}$ measurable, $p \geq 1)$ whenever Lebesgue measure $d x$ is understood.

$\left[L^{2}((0, \ell))\right]^{r}$ is the class of square integrable vector functions on $(0, \ell)$ with values in $\mathbb{C}^{r}$ and scalar product

$$
(f, g)=\int_{0}^{\ell} g(x)^{*} f(x) d x=\sum_{s=1}^{r} \int_{0}^{\ell} \overline{g_{s}(x)} f_{s}(x) d x,
$$

where $f=\left(f_{1}, \ldots, f_{r}\right)^{\top}, g=\left(g_{1}, \ldots, g_{r}\right)^{\top} \in\left[L^{2}((0, \ell))\right]^{r}(r \in \mathbb{N})$.

Moreover, $\mathcal{B}(\mathcal{H})$ denotes the class of bounded linear operators, which map the Hillbert space $\mathcal{H}$ into itself. Bounded operators which map the Hilbert space $\mathcal{H}_{1}$ into the Hilbert space $\mathcal{H}_{2}$ are denoted by $\mathcal{B}\left(\mathcal{H}_{1}, \mathcal{H}_{2}\right)$.

By $C^{k}(\Omega)$ we denote the usual class of functions on the open set $\Omega \subseteq \mathbb{R}^{n}$ for some $n \in \mathbb{N}$, whose partial derivatives up to order $k$ are continuous, and $C(\Omega)$ stands for continuous functions on $\Omega$. Similarly, $C^{k}(\bar{\Omega})$ denotes the space of functions whose partial derivatives up to order $k$ are bounded and uniformly continuous on $\Omega$ (see, e.g., [1, Ch. 1]). When functions belong to $C^{k}(\Omega)$, we say that they are $k$ times continuously differentiable.

We employ the notation $\mathbb{C}_{+}=\{z \in \mathbb{C} \mid \operatorname{Im}(z)>0\}$ for the open complex upper half-plane. 


\section{Preliminaries}

Fundamental solutions of (1.6) play an essential role in this theory (it is always assumed that the entries of $v$ are locally integrable on $[0, \infty)$ and so the fundamental solutions exist). See Notation 1.1 and formula (1.8) for the normalizations of the considered fundamental solutions. In view of (1.8) one has the equality

$$
u_{0}(x, z)=u_{\ell}(x, z) u_{0}(\ell, z) .
$$

Taking into account Definition 1.2 and formula (2.1) one derives

$$
u_{\ell}(\cdot, z) u_{0}(\ell, z)\left[\begin{array}{c}
I_{m_{1}} \\
\varphi_{0}(z)
\end{array}\right] \in\left[L^{2}((0, \infty))\right]^{m \times m_{1}} .
$$

According to the representation [28, eq. (2.26)] of $\varphi_{0}(z)$ (see [28, Cor. 2.21]) and by the corresponding formula (2.29) in [28, one obtains

$$
\left[\begin{array}{ll}
I_{m_{1}} & \varphi_{0}(z)^{*}
\end{array}\right] u_{0}(\ell, z)^{*} J u_{0}(\ell, z)\left[\begin{array}{c}
I_{m_{1}} \\
\varphi_{0}(z)
\end{array}\right] \geq 0_{m_{1}} .
$$

Next, similarly to (1.7), we now partition $u_{0}(\ell, z)$ into four blocks: $u_{0}(\ell, z)=$ $\left\{u_{0, j k}(\ell, z)\right\}_{j, k=1}^{2}$. Then formula (2.3) yields the fact

$$
\operatorname{det}\left(u_{0,11}(\ell, z)+u_{0,12}(\ell, z) \varphi_{0}(z)\right) \neq 0 .
$$

Therefore, one obtains

$$
\begin{aligned}
& u_{0}(\ell, z)\left[\begin{array}{c}
I_{m_{1}} \\
\varphi_{0}(z)
\end{array}\right]=\left[\begin{array}{c}
I_{m_{1}} \\
\widetilde{\varphi}_{\ell}(z)
\end{array}\right]\left(u_{0,11}(\ell, z)+u_{0,12}(\ell, z) \varphi_{0}(z)\right), \\
& \widetilde{\varphi}_{\ell}(z):=\left(u_{0,21}(\ell, z)+u_{0,22}(\ell, z) \varphi_{0}(z)\right)\left(u_{0,11}(\ell, z)+u_{0,12}(\ell, z) \varphi_{0}(z)\right)^{-1} .
\end{aligned}
$$

Formulas (2.2), (2.4), and (2.5) imply that

$$
u_{\ell}(\cdot, z)\left[\begin{array}{c}
I_{m_{1}} \\
\widetilde{\varphi}_{\ell}(z)
\end{array}\right] \in\left[L^{2}((\ell, \infty))\right]^{m \times m_{1}} .
$$

Comparing (1.9) and (2.7), and taking into account that the Weyl-Titchmarsh function is unique, one infers that $\varphi_{\ell}(z)=\widetilde{\varphi}_{\ell}(z)$, and hence (2.6) takes on the form

$$
\varphi_{\ell}(z)=\left(u_{0,21}(\ell, z)+u_{0,22}(\ell, z) \varphi_{0}(z)\right)\left(u_{0,11}(\ell, z)+u_{0,12}(\ell, z) \varphi_{0}(z)\right)^{-1} .
$$

Theorem 2.1. Suppose that $v \in\left[L^{1}((0, R))\right]^{m_{1} \times m_{2}}$ for all $R>0$ and assume that $u_{0}(\ell, z)=\left\{u_{0, j k}(\ell, z)\right\}_{j, k=1}^{2}$ represents the value of the fundamental solution $u_{0}$ of the system (1.6) at the point $x=\ell$. Then the Weyl-Titchmarsh functions $\varphi_{\ell}$ and $\varphi_{0}$ of the system (1.6) on the semi-axes $[\ell, \infty)$ and $[0, \infty)$, respectively, are connected by relation (2.8).

Proposition 2.2. Let some $m \times m$ matrix function $w(x)$ satisfy the differential equation $w^{\prime}(x)=G(x) w(x)$, where $G \in\left[L^{1}((0, R))\right]^{m \times m}$ for all $R>0$ has the block form $G=\left\{G_{j k}\right\}_{j, k=1,2}^{2}$ and $G_{j j}$ is an $m_{j} \times m_{j}$ matrix-valued function ( $j=$ 1,2). Let $P$ and $Q$ be $m_{1} \times m_{1}$ and $m_{2} \times m_{1}$ matrices, respectively, and suppose that $\operatorname{det}\left(G_{11}(x) P+G_{12}(x) Q\right) \neq 0$ holds on some open subset $\Omega \subseteq \mathbb{R}$. Then (for a.e. $x \in \Omega$ ), the linear fractional transformation

$$
\phi(x)=\left(G_{21}(x) P+G_{22}(x) Q\right)\left(G_{11}(x) P+G_{12}(x) Q\right)^{-1}
$$


satisfies the following matrix-valued Riccati-type differential equation (for a.e. $x \in$ $\Omega)$

$$
\phi^{\prime}(x)=-\phi(x) G_{12}(x) \phi(x)-\phi(x) G_{11}(x)+G_{22}(x) \phi(x)+G_{21}(x) .
$$

In view of (1.9) and (2.8), we rewrite (2.10), for the case of the Weyl-Titchmarsh function $\varphi_{\ell}(z)$, in the form,

$$
\frac{d}{d \ell} \varphi_{\ell}(z)=-i\left(\varphi_{\ell}(z) v(\ell) \varphi_{\ell}(z)+v(\ell)^{*}+2 z \varphi_{\ell}(z)\right) .
$$

\section{The $\mathcal{A}$-Function for Dirac-Type Systems: Part I}

Analogs of the $\mathcal{A}$-function for Dirac-type systems were considered in [26] for the case $m_{1}=m_{2}$ (see also some related results in [25]). The case of rectangular potentials $v$ was dealt with in [8,27,28].

In order to construct the $\mathcal{A}$-equation for Dirac-type system we need an analog of the $A$-function representation (1.2) of the Weyl-Titchmarsh function $\varphi$. The results were developed further in [26] and for the rectangular $m_{2} \times m_{1}$ Weyl-Titchmarsh functions in [8,27.

In this paragraph, we present some results from 27. (more precisely, Proposition 3.1, Theorem 4.1, and Corollary 4.2 in 27) that we need for our considerations. We recall that $u_{0}(x, z)$ is the fundamental solution of system (1.6) and now partition $u_{0}(x, 0)$ into block rows

$$
\begin{aligned}
& \beta(x)=\left[\begin{array}{ll}
\beta_{1}(x) & \beta_{2}(x)
\end{array}\right]:=\left[\begin{array}{ll}
I_{m_{1}} & 0_{m_{1} \times m_{2}}
\end{array}\right] u_{0}(x, 0), \\
& \gamma(x)=\left[\begin{array}{ll}
\gamma_{1}(x) & \gamma_{2}(x)
\end{array}\right]:=\left[\begin{array}{ll}
0_{m_{2} \times m_{1}} & I_{m_{2}}
\end{array}\right] u_{0}(x, 0),
\end{aligned}
$$

where $\beta_{1}$ and $\gamma_{2}$ are $m_{1} \times m_{1}$ and $m_{2} \times m_{2}$ matrix-valued functions, respectively. In view of (1.6) one obtains

$$
\gamma^{\prime}(x)=-i v(x)^{*} \beta(x) .
$$

The system (1.6) also yields $u_{0}(x, 0)^{*} J u_{0}(x, 0)=J=u_{0}(x, 0) J u_{0}(x, 0)^{*}$. In particular, one has

$$
\gamma(x) J \gamma(x)^{*} \equiv-I_{m_{2}}, \quad \beta(x) J \gamma(x)^{*} \equiv 0_{m_{1} \times m_{2}} .
$$

From (3.3) and the second equality in (3.4) it follows that

$$
\gamma^{\prime}(x) J \gamma(x)^{*} \equiv 0_{m_{2}} .
$$

The following operators $K$, acting in $\left[L^{2}((0, T))\right]^{m_{2}}(T \in(0, \infty))$,

$$
(K f)(x)=i \gamma(x) \int_{0}^{x} J \gamma(t)^{*} f(t) d t, \quad f \in\left[L^{2}((0, T))\right]^{m_{2}},
$$

play an essential role in solving the inverse problem of recovering $v$ from the WeylTitchmarsh function $\varphi$.

Proposition 3.1. Suppose that $v \in\left[L^{2}((0, T))\right]^{m_{1} \times m_{2}}(T \in(0, \infty))$ in the Diractype system (1.6), and let $K$ be given by (3.6), where $\gamma$ is defined in (3.2). Then 
there is a similarity transformation operator $E \in \mathcal{B}\left(\left[L^{2}((0, T))\right]^{m_{2}}\right)$ such that

$$
\begin{aligned}
& K=E \mathcal{I} E^{-1}, \quad(\mathcal{I} f)(x):=-i \int_{0}^{x} f(t) d t \quad(x>0), \\
& (E f)(x)=f(x)+\int_{0}^{x} N(x, t) f(t) d t, \quad f \in\left[L^{2}((0, T))\right]^{m_{2}} \\
& \left(E^{-1} \gamma_{2}\right)(x) \equiv I_{m_{2}} \quad(x>0),
\end{aligned}
$$

where $N(\cdot, \cdot)$ is a Hilbert-Schmidt integral kernel, $E^{-1}$ is applied to $\gamma_{2}(x)$ in (3.9) columnwise, and (3.9) has to be understood in the following way: the image of the $k$-th column of $\gamma_{2}(x)$ equals the constant vector function given by the $k$-th column of $I_{m_{2}}\left(1 \leq k \leq m_{2}\right)$. Moreover, the operators $E^{ \pm 1}$ map differentiable vectorvalued functions with a square integrable derivative into vector-valued functions of the same class.

Remark 3.2. Formulas similar to (3.9) will also appear in the remainder of this paper and we understand them in the analogous manner: namely, operators are applied to matrix functions columnwise, and $I_{m_{2}}$ in this context is understood as the corresponding constant matrix function, which takes the values $I_{m_{2}}$ at each $x$.

Remark 3.3. The operator $E$ satisfying the conditions of Proposition 3.1 is constructed in the proof of [27, Proposition 3.1] as a product $E=\widetilde{E} E_{0}$, where $\widetilde{E}$ satisfies all the conditions of Proposition 3.1 excluding, possibly, (3.9) and

$$
\begin{aligned}
& \left(E_{0} f\right)(x)=f(x)+\int_{0}^{x} N_{0}(x-t) f(t) d t, \quad f \in\left[L^{2}((0, T))\right]^{m_{2}}, \\
& N_{0}(x):=\left(\widetilde{E}^{-1} \gamma_{2}\right)^{\prime}(x) \quad(\text { for a.e. } x \in(0, T)) .
\end{aligned}
$$

More precisely, there is a whole family of triangular operators satisfying all the conditions of Proposition 3.1 excluding, possibly, (3.9), and $\widetilde{E}$ is one of them. The construction of the similarity transformation operator $\widetilde{E}$ is based on the work [29] and uses the resolvent of $K$. We normalize $\widetilde{E}$ using the triangular convolution operator $E_{0}$ so that (3.9) holds for $E=\widetilde{E} E_{0}$. (One notes that $E_{0}$ depends on $\widetilde{E}$, see the definition of $N_{0}$ in (3.10).)

Using the operator $E$ we now introduce the $m_{2} \times m_{1}$ matrix-valued function

$$
\Phi(x):=\left(E^{-1} \gamma_{1}\right)(x) \quad(0<x<T) .
$$

According to Proposition 3.1, $\Phi$ is differentiable and, in the case of locally bounded potentials $v$ (see [28, eq. (2.154)]) the following equality holds:

$$
v(x)=\left(i E \Phi^{\prime}\right)(x)^{*} \quad(0<x<T) .
$$

Remark 3.4. We note that, by construction, the function $N(x, t)$ in (3.8) does not depend (for any fixed $T_{0}>0$ and values $x<T_{0}$ ) on the choice of $T \geq T_{0}$. This means that (3.11) (for various values of $T$ ) uniquely determines $\Phi(x)$ on the whole half-axis $(0, \infty)$. Taking into account [27. Proposition 1.3], we now reformulate Theorem 4.1 and Corollary 4.2 in [27] in the following manner. 
Proposition 3.5. Let $\varphi \equiv \varphi_{0}$ be the Weyl-Titchmarsh function of the Dirac system (1.6) on $[0, \infty)$, assuming $v \in\left[L^{2}((0, R))\right]^{m_{1} \times m_{2}}$ for all $R>0$. Then (uniformly with respect to $\operatorname{Re}(z))$ one has

$$
\varphi(z) \underset{\operatorname{Im}(z) \rightarrow \infty}{=} 2 i z \int_{0}^{T} e^{2 i x z} \Phi(x) d x+O\left(z e^{2 i T z} /(\operatorname{Im}(z))^{1 / 2}\right),
$$

implying

$$
\varphi(z)=2 i z \int_{0}^{\infty} e^{2 i x z} \Phi(x) d x \quad(\operatorname{Im}(z)>0) .
$$

Remark 3.6. Formula (3.13) is an analog of equality (1.2) and the $\mathcal{A}$-equation for Dirac systems will be formulated in terms of $\Phi$ and its derivatives. In order to use (3.13) or (3.14) and rewrite (2.11) in terms of $\Phi$ as an $\mathcal{A}$-equation, we should integrate the integrals in (3.13) or (3.14) by parts. Therefore, $\Phi(x)$ should be two times differentiable.

\section{The $\mathcal{A}$-Function for Dirac-Type Systems: Part II}

In this section we consider the case $v \in\left[C^{1}([0, \infty))\right]^{m_{1} \times m_{2}}$. Our next proposition deals with the differentiability of $\Phi$ mentioned at the end of Remark 3.6 .

Proposition 4.1. Assume that $v \in\left[C^{1}([0, \infty))\right]^{m_{1} \times m_{2}}$ in the Dirac-type system (1.6). Then (cf. (3.11) $)$

$$
\Phi(x) \in\left[C^{2}([0, \infty))\right]^{m_{2} \times m_{1}} .
$$

Proof. We divide the proof into four steps.

Step 1. We consider the operator $\widetilde{E}$ in $\left[L^{2}((0, T))\right]^{m_{2}}$ given by

$$
(\widetilde{E} f)(x)=f(x)+\int_{0}^{x} \tilde{N}(x, t) f(t) d t, \quad f \in\left[L^{2}((0, T))\right]^{m_{2}},
$$

(for a.e. $x \in(0, T)$ ) discussed in Remark 3.3. In view of Proposition 3.1 and Remark 3.3 one has

$$
K=\widetilde{E} \mathcal{I} \widetilde{E}^{-1} .
$$

Next, we show that $\widetilde{E}^{-1}$ maps vector-valued $C^{2}([0, T])^{m_{2}}$ functions into vectorvalued functions of the same class.

Assuming $v \in\left[C^{1}([0, \infty))\right]^{m_{1} \times m_{2}}$, the construction of $\widetilde{N}(\cdot, \cdot)$ in [27, Lemma 2.3] then implies that $\widetilde{N}(x, t)$ in (4.2) is continuous on the domain $0 \leq t \leq x<\infty$, and that

$$
\widetilde{N}(x, 0)=0_{m_{2}} .
$$

Since $\widetilde{N}(x, t)$ is continuous, the integral kernel $\widetilde{N}^{\times}(x, t)$ of the operator

$$
\left(\widetilde{E}^{-1} f\right)(x)=f(x)+\int_{0}^{x} \widetilde{N}^{\times}(x, t) f(t) d t, \quad f \in\left[L^{2}((0, T))\right]^{m_{2}}
$$

(for $x \in(0, T)$ ), is continuous as well. Moreover, using (4.2) and (4.5) in order to detail the integral kernel of $\widetilde{E}^{-1} \widetilde{E}$, one obtains

$$
\widetilde{N}^{\times}(x, t)+\widetilde{N}(x, t)+\int_{t}^{x} \widetilde{N}^{\times}(x, r) \widetilde{N}(r, t) d r=0_{m_{2}} .
$$


Clearly, (4.4) and (4.6) yield

$$
\tilde{N}^{\times}(x, 0)=0_{m_{2}} .
$$

We shall need (4.6) and (4.7) in future considerations, but first we consider $K$ in greater detail. Clearly, $v \in\left[C^{1}([0, \infty))\right]^{m_{1} \times m_{2}}$ implies that $\gamma \in\left[C^{2}([0, \infty))\right]^{m_{2} \times m}$. It is immediate from (3.6) that (for $x \in(0, T)$ )

$$
\begin{aligned}
& (K f)^{\prime}(x)=i \gamma(x) J \gamma(x)^{*} f(x)+i \gamma^{\prime}(x) \int_{0}^{x} J \gamma(t)^{*} f(t) d t, \\
& (K f)(0)=0_{m_{2} \times 1}, \quad f \in\left[L^{2}((0, T))\right]^{m_{2}} .
\end{aligned}
$$

Taking into account (3.4) and (4.8), one rewrites $K$ in the form (for $x \in(0, T)$ )

$$
\begin{aligned}
& (K f)(x)=i \mathcal{I}(K f)^{\prime}(x)=\left(\mathcal{I}\left(I_{\left[L^{2}((0, T))\right]^{m_{2}}}-K_{1}\right) f\right)(x), \\
& \left(K_{1} f\right)(x):=\gamma^{\prime}(x) \int_{0}^{x} J \gamma(t)^{*} f(t) d t, \quad f \in\left[L^{2}((0, T))\right]^{m_{2}} .
\end{aligned}
$$

In particular, $K=\mathcal{I}\left(I_{\left[L^{2}((0, T))\right]^{m_{2}}}-K_{1}\right)$. Rewriting (4.3) and using (4.9), one arrives at

$$
\widetilde{E}^{-1} K=\mathcal{I} \widetilde{E}^{-1}, \quad \widetilde{E}^{-1} \mathcal{I}=\mathcal{I} \widetilde{E}^{-1}\left(I_{\left[L^{2}((0, T))\right]^{m_{2}}}-K_{1}\right)^{-1} .
$$

Step 2. We denote the integral kernels of the operators $\left(I_{\left[L^{2}((0, T))\right]^{m_{2}}}-K_{1}\right)$ and $\left(I_{\left[L^{2}((0, T))\right]^{m_{2}}}-K_{1}\right)^{-1}$ by $-N_{1}$ and by $-N_{1}^{\times}$, respectively. That is, we set (for $x \in(0, T))$

$$
\begin{aligned}
& \left(K_{1} f\right)(x)=\int_{0}^{x} N_{1}(x, t) f(t) d t, \quad N_{1}(x, t)=\gamma^{\prime}(x) J \gamma(t)^{*}, \\
& \left(\left(I_{\left[L^{2}((0, T))\right]^{m_{2}}}-K_{1}\right)^{-1} f\right)(x)=f(x)-\int_{0}^{x} N_{1}^{\times}(x, t) f(t) d t, \quad f \in\left[L^{2}((0, T))\right]^{m_{2}} .
\end{aligned}
$$

One recalls that $\gamma \in\left[C^{2}([0, \infty))\right]^{m_{2} \times m}$, and so $N_{1}(\cdot, \cdot)$ (given in (4.11)) is differentiable with a continuous derivative. By virtue of (3.5) and (4.11) one obtains $N_{1}(x, x)=0_{m_{2}}$.

Since $N_{1}(\cdot, \cdot)$ is continuous, $N_{1}^{\times}(\cdot, \cdot)$ is continuous as well. In order to show that $N_{1}^{\times}(\cdot, \cdot)$ is differentiable, we consider the integral kernel of $\left(I_{\left[L^{2}((0, T))\right]^{m_{2}}}-\right.$

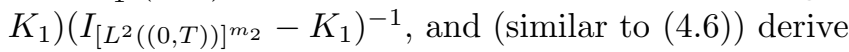

$$
N_{1}^{\times}(x, t)+N_{1}(x, t)=\int_{t}^{x} N_{1}(x, r) N_{1}^{\times}(r, t) d r .
$$

Taking into account that the derivatives of $N_{1}$ are continuous and using (4.13), one concludes that $\frac{\partial}{\partial x} N_{1}^{\times}(x, t)$ is continuous. In view of the relations (4.13) and $N_{1}(x, x)=0_{m_{2}}$, one gets $N_{1}^{\times}(x, x)=0_{m_{2}}$. Hence, we next introduce the operator $K_{2}$ in $\left[L^{2}((0, T))\right]^{m_{2}}$ by the equalities (for $x \in(0, T)$ )

$$
\begin{array}{r}
\left(K_{2} f\right)(x)=\frac{d}{d x} \int_{0}^{x} N_{1}^{\times}(x, t) f(t) d t=\int_{0}^{x}\left(\frac{\partial}{\partial x} N_{1}^{\times}\right)(x, t) f(t) d t, \\
f \in\left[L^{2}((0, T))\right]^{m_{2}} .
\end{array}
$$


Relations (4.12) and (4.14) then yield the following representation of $\left(I_{\left[L^{2}((0, T))\right]^{m_{2}}}-\right.$ $\left.K_{1}\right)^{-1}$ :

$$
\begin{aligned}
& \left(I_{\left[L^{2}((0, T))\right]^{m_{2}}}-K_{1}\right)^{-1}=I_{\left[L^{2}((0, T))\right]^{m_{2}}}-i \mathcal{I} K_{2}, \\
& \left(K_{2} f\right)(x)=\int_{0}^{x} N_{2}(x, t) f(t) d t, \quad f \in\left[L^{2}((0, T))\right]^{m_{2}}, \\
& N_{2}(x, t)=\left(\frac{\partial}{\partial x} N_{1}^{\times}\right)(x, t), \quad(x, t) \in[0, T] \times[0, T],
\end{aligned}
$$

where $N_{2}(\cdot, \cdot)$ is continuous on $[0, T] \times[0, T]$. From (4.10) and (4.15) one infers that

$$
\widetilde{E}^{-1} \mathcal{I}=\mathcal{I} \widetilde{E}^{-1}-i \mathcal{I} \widetilde{E}^{-1} \mathcal{I} K_{2},
$$

and the substitution of (4.10) into the right-hand side of this equality results in

$$
\widetilde{E}^{-1} \mathcal{I}=\mathcal{I} \widetilde{E}^{-1}-i \mathcal{I}^{2} \widetilde{E}^{-1}\left(I_{\left[L^{2}((0, T))\right]^{m_{2}}}-K_{1}\right)^{-1} K_{2} .
$$

Right-multiplying both sides of (4.17) by $\mathcal{I}$ one gets

$$
\widetilde{E}^{-1} \mathcal{I}^{2}=\mathcal{I} \widetilde{E}^{-1} \mathcal{I}-i \mathcal{I}^{2} \widetilde{E}^{-1}\left(I_{\left[L^{2}((0, T))\right]^{m_{2}}}-K_{1}\right)^{-1} K_{2} \mathcal{I} .
$$

Substituting (4.17) into the right-hand side of (4.18) one derives

$$
\begin{aligned}
\widetilde{E}^{-1} \mathcal{I}^{2}= & \mathcal{I}\left(\mathcal{I} \widetilde{E}^{-1}-i \mathcal{I}^{2} \widetilde{E}^{-1}\left(I_{\left[L^{2}((0, T))\right]^{m_{2}}}-K_{1}\right)^{-1} K_{2}\right) \\
& -i \mathcal{I}^{2} \widetilde{E}^{-1}\left(I_{\left.\left[L^{2}((0, T))\right]^{m_{2}}-K_{1}\right)^{-1} K_{2} \mathcal{I}}\right. \\
= & \mathcal{I}^{2}\left(\widetilde{E}^{-1}-i \mathcal{I} \widetilde{E}^{-1}\left(I_{\left[L^{2}((0, T))\right]^{m_{2}}}-K_{1}\right)^{-1} K_{2}\right. \\
& \left.-i \widetilde{E}^{-1}\left(I_{\left[L^{2}((0, T))\right]^{m_{2}}}-K_{1}\right)^{-1} K_{2} \mathcal{I}\right) .
\end{aligned}
$$

Finally, we note that any $f \in\left[C^{2}([0, T])\right]^{m_{2}}$ admits the representation

$$
f(x)=f(0)+i \mathcal{I} f^{\prime}(0)-\left(\mathcal{I}^{2} f^{\prime \prime}\right)(x) .
$$

Recalling that $\mathcal{I}$ is the integration operator (multiplied by $-i$ ) and taking into account (4.17)- (4.20) one concludes that $\widetilde{E}^{-1} f \in\left[C^{2}([0, T])\right]^{m_{2}}$ for any $f \in$ $\left[C^{2}([0, T])\right]^{m_{2}}$ if only $\left(\widetilde{E}^{-1} f(0)\right)(x)$ belongs $\left[C^{2}([0, T])\right]^{m_{2}}$ for any constant vector function $f(0)$. Recalling Remark 3.2 we rewrite this condition as

$$
\left(\widetilde{E}^{-1} I_{m_{2}}\right)(x) \in\left[C^{2}([0, T])\right]^{m_{2} \times m_{2}} .
$$

Step 3. We rewrite the equality $\widetilde{E}^{-1} K=\mathcal{I} \widetilde{E}^{-1}$ in (4.10) in terms of the integral kernels of the corresponding operators:

$$
I_{m_{2}}+\gamma(x) J \gamma(t)^{*}+\int_{t}^{x} \tilde{N}^{\times}(r, t) d r+\int_{t}^{x} \tilde{N}^{\times}(x, r) \gamma(r) d r J \gamma(t)^{*}=0_{m_{2}} .
$$

Setting $t=0$ in (4.22) and recalling (4.7), one obtains

$$
\widetilde{E}^{-1}\left(-\gamma(x) J \gamma(0)^{*}\right)=I_{m_{2}} .
$$

Formulas (3.4), (3.5) and (4.20) imply that

$$
-\gamma(x) J \gamma(0)^{*}=I_{m_{2}}+\mathcal{I}^{2} \gamma^{\prime \prime}(x) J \gamma(0)^{*} .
$$

It is immediate from (4.19) that

$$
\widetilde{E}^{-1} \mathcal{I}^{2} \gamma^{\prime \prime}(x) J \gamma(0)^{*} \in\left[C^{2}([0, T])\right]^{m_{2} \times m_{2}},
$$


and (4.21) follows from (4.23)- (4.25). This completes the proof that $\widetilde{E}^{-1} f \in$ $\left[C^{2}([0, T])\right]^{m_{2}}$ for any $f \in\left[C^{2}([0, T])\right]^{m_{2}}$.

Step 4. One recalls that relations (3.11) (varying $T \in(0, \infty)$ ) uniquely determine $\Phi$ on $[0, \infty)$ and that according to Remark 3.3 one has $E=\widetilde{E} E_{0}$ for $E$ in (3.11). Thus, in order to prove Proposition 4.1 it remains to show that

$$
E_{0}^{-1} f \in\left[C^{2}([0, T])\right]^{m_{2}} \text { for any } f \in\left[C^{2}([0, T])\right]^{m_{2}},
$$

where $E_{0}$ has the form (3.10). It is easy to see (and is used in the proof of [27, Proposition 3.1]) that

$$
E_{0}^{-1} \mathcal{I}=\mathcal{I} E_{0}^{-1} .
$$

In view of (4.20) and (4.27), we (similarly to the case of $\left.\widetilde{E}^{-1}\right)$ see that $\left(E_{0}^{-1} f\right)(x) \in$ $\left[C^{2}([0, T])\right]^{m_{2}}$ if $E_{0}^{-1}$ applied to the constant vector $f(0)$ belongs $\left[C^{2}([0, T])\right]^{m_{2}}$. In other words, it remains to show that $E_{0}^{-1} I_{m_{2}}$ belongs $\left[C^{2}([0, T])\right]^{m_{2} \times m_{2}}$.

In order to prove $\left(E_{0}^{-1} I_{m_{2}}\right)(x) \in\left[C^{2}([0, T])\right]^{m_{2} \times m_{2}}$, one notes that according to (3.10) one has

$$
\begin{aligned}
\left(E_{0} I_{m_{2}}\right)(x) & =I_{m_{2}}+\int_{0}^{x} N_{0}(t) d t=I_{m_{2}}+i \mathcal{I}\left(\left(\widetilde{E}^{-1} \gamma_{2}\right)^{\prime}(x)\right) \\
& =I_{m_{2}}+i \mathcal{I}\left(\left(\widetilde{E}^{-1} \gamma_{2}\right)^{\prime}(0)+i \mathcal{I}\left(\widetilde{E}^{-1} \gamma_{2}\right)^{\prime \prime}(x)\right)
\end{aligned}
$$

which (using (4.27) ) may be rewritten in the form

$$
\left(E_{0}^{-1} I_{m_{2}}\right)(x)=I_{m_{2}}-i \mathcal{I} E_{0}^{-1}\left(\left(\widetilde{E}^{-1} \gamma_{2}\right)^{\prime}(0)+i \mathcal{I}\left(\widetilde{E}^{-1} \gamma_{2}\right)^{\prime \prime}(x)\right) .
$$

Clearly, the right-hand side of (4.29) belongs to $\left[C^{1}([0, T])\right]^{m_{2} \times m_{2}}$, and so $\left(E_{0}^{-1} I_{m_{2}}\right)(x)$ belongs to $\left[C^{1}([0, T])\right]^{m_{2} \times m_{2}}$. Hence, $E_{0}^{-1}\left(\widetilde{E}^{-1} \gamma_{2}\right)^{\prime}(0)$ belongs to $\left[C^{1}([0, T])\right]^{m_{2} \times m_{2}}$, which implies that the right-hand side of (4.29) belongs to $\left[C^{2}([0, T])\right]^{m_{2} \times m_{2}}$, and the required relation $\left(E_{0}^{-1} I_{m_{2}}\right)(x) \in\left[C^{2}([0, T])\right]^{m_{2} \times m_{2}}$ follows.

Remark 4.2. Together with the system (1.6) one can consider the shifted systems

$$
\widehat{y}_{\ell}^{\prime}(x, z)=i\left(z J+J \widehat{V}_{\ell}(x)\right) \widehat{y}_{\ell}(x, z), \quad \widehat{V}_{\ell}(x):=V(x+\ell) \quad(x \geq 0),
$$

where $\ell \geq 0$ and the fundamental solution is denoted by $\widehat{u}_{\ell}(x, z)$. It is easy to see that

$$
\widehat{u}_{\ell}(x, z)=u_{\ell}(x+\ell, z) .
$$

Equality (4.31) and Definition 1.2 imply that $\varphi_{\ell}(z)$ is the Weyl-Titchmarsh function of the system (4.30).

Notation 4.3. Henceforth, we introduce the additional parameter $\ell$ in our notation of the corresponding systems (4.30) (instead of the system (1.6)) and write, for instance, $\beta(x, \ell), \gamma(x, \ell), \Phi(x, \ell)$, etc. In addition, we set

$$
\mathcal{A}(x, \ell):=\frac{\partial}{\partial x} \Phi(x, \ell) \equiv \Phi^{\prime}(x, \ell) .
$$


Clearly, (3.12) holds pointwise in the case $v \in\left[C^{1}([0, \infty))\right]^{m_{1} \times m_{2}}$. Formulas (3.8) and (3.12), together with the equalities $\widehat{V}_{\ell}(x):=V(x+\ell)$ and (4.32) above, imply that

$$
v(0)=-i \Phi^{\prime}(0)^{*}, \quad v(\ell)=-i \Phi^{\prime}(0, \ell)^{*}=-i \mathcal{A}(0, \ell)^{*} .
$$

\section{The $\mathcal{A}$-Equation for Dirac-Type Systems}

Using (3.13) and (4.33) we now rewrite (2.11) in the form of the $\mathcal{A}$-equation for Dirac-type systems and obtain the following result.

Theorem 5.1. Suppose that $v \in\left[C^{1}([0, \infty))\right]^{m_{1} \times m_{2}}$ in the Dirac-type system (1.6). Then the $\mathcal{A}(\cdot, \cdot)$-function given by (4.32) is jointly continuously differentiable in both variables, $\mathcal{A}(\cdot, \cdot) \in\left[C^{1}([0, \infty) \times[0, \infty))\right]^{m_{2} \times m_{1}}$, and satisfies the integro-differential equation $($ for $(x, \ell) \in[0, \infty) \times[0, \infty))$

$$
\frac{\partial}{\partial \ell} \mathcal{A}(x, \ell)=\frac{\partial}{\partial x} \mathcal{A}(x, \ell)+\int_{0}^{x} \mathcal{A}(x-t, \ell) \mathcal{A}(0, \ell)^{*} \mathcal{A}(t, \ell) d t .
$$

Proof. We divide the proof into two steps.

Step 1. Formula (3.11) implies that

$$
\Phi(0)=\left(E^{-1} \gamma_{1}\right)(0)=\gamma_{1}(0)=0_{m_{2} \times m_{1}} .
$$

In view of (3.13), (4.32) and (5.2) one infers that

$$
\begin{aligned}
\varphi_{\ell}(z) \underset{\operatorname{Im}(z) \rightarrow \infty}{=} \int_{0}^{T} \Phi(x, \ell) d\left(e^{2 i x z}\right)+O\left(z e^{2 i T z} /(\operatorname{Im}(z))^{1 / 2}\right) \\
\underset{\operatorname{Im}(z) \rightarrow \infty}{=}-\int_{0}^{T} e^{2 i x z} \mathcal{A}(x, \ell) d x+O\left(z e^{2 i T z} /(\operatorname{Im}(z))^{1 / 2}\right) .
\end{aligned}
$$

According to Proposition 4.1, $\mathcal{A}(x, \ell)$ is continuously differentiable with respect to $x$. Hence, taking into account (4.33), one rewrites (4.30) in the form

$$
\begin{gathered}
2 z \varphi_{\ell}(z) \underset{\operatorname{Im}(z) \rightarrow \infty}{=}-i \mathcal{A}(0, \ell)-i \int_{0}^{T} e^{2 i x z} \mathcal{A}^{\prime}(x, \ell) d x+O\left(z^{2} e^{2 i T z} /(\operatorname{Im}(z))^{1 / 2}\right) \\
\operatorname{Im}(z) \rightarrow \infty \\
=-v(\ell)^{*}-i \int_{0}^{T} e^{2 i x z} \mathcal{A}^{\prime}(x, \ell) d x+O\left(z^{2} e^{2 i T z} /(\operatorname{Im}(z))^{1 / 2}\right) .
\end{gathered}
$$

Moreover, using (5.3) and (4.33), the representation of $\varphi_{\ell}(z) v(\ell) \varphi_{\ell}(z)$ reads as follows

$$
\begin{aligned}
\varphi_{\ell}(z) v(\ell) \varphi_{\ell}(z) \underset{\operatorname{Im}(z) \rightarrow \infty}{=} & -i \int_{0}^{T} \int_{0}^{T} e^{2 i(\tau+t) z} \mathcal{A}(\tau, \ell) d \tau \mathcal{A}(0, \ell)^{*} \mathcal{A}(t, \ell) d t \\
& +O\left(z e^{2 i T z} /(\operatorname{Im}(z))^{1 / 2}\right) \\
\operatorname{Im}(z) \rightarrow \infty & -i \int_{0}^{T} \int_{t}^{T+t} e^{2 i x z} \mathcal{A}(x-t, \ell) d x \mathcal{A}(0, \ell)^{*} \mathcal{A}(t, \ell) d t \\
& +O\left(z e^{2 i T z} /(\operatorname{Im}(z))^{1 / 2}\right)
\end{aligned}
$$


Changing the order of integration in (5.5) (and removing the swiftly decaying part in the result) one arrives at

$$
\begin{aligned}
\varphi_{\ell}(z) v(\ell) \varphi_{\ell}(z) \underset{\operatorname{Im}(z) \rightarrow \infty}{=} & -i \int_{0}^{T} e^{2 i x z} \int_{0}^{x} \mathcal{A}(x-t, \ell) \mathcal{A}(0, \ell)^{*} \mathcal{A}(t, \ell) d t d x \\
& +O\left(z e^{2 i T z} /(\operatorname{Im}(z))^{1 / 2}\right) .
\end{aligned}
$$

Taking into account (5.4) and (5.6) one rewrites (2.11) in the form

$$
\begin{aligned}
\frac{d}{d \ell} \varphi_{\ell}(z) \underset{\operatorname{Im}(z) \rightarrow \infty}{=} & -\int_{0}^{T} e^{2 i x z} \int_{0}^{x} \mathcal{A}(x-t, \ell) \mathcal{A}(0, \ell)^{*} \mathcal{A}(t, \ell) d t d x \\
& -\int_{0}^{T} e^{2 i x z} \mathcal{A}^{\prime}(x, \ell) d x+O\left(z^{2} e^{2 i T z} /(\operatorname{Im}(z))^{1 / 2}\right) .
\end{aligned}
$$

Step 2. A considerable part of the proof connected with the representation of $\frac{d}{d \ell} \varphi_{\ell}(z)$ is moved to Appendix A. Assuming temporarily that $\operatorname{supp}(v) \subseteq[0, a]$, one derives with the help of (5.7) and (A.75),

$$
\begin{aligned}
& \int_{0}^{T} e^{2 i x z} \mathcal{F}(x, \ell) d x \underset{\operatorname{Im}(z) \rightarrow \infty}{=} O\left(z^{2} e^{2 i T z} /(\operatorname{Im}(z))^{1 / 2}\right), \\
& \mathcal{F}(x, \ell):=\frac{\partial}{\partial \ell} \mathcal{A}(x, \ell)-\frac{\partial}{\partial x} \mathcal{A}(x, \ell)-\int_{0}^{x} \mathcal{A}(x-t, \ell) \mathcal{A}(0, \ell)^{*} \mathcal{A}(t, \ell) d t .
\end{aligned}
$$

It is immediate from (5.8) that

$$
\Upsilon(z, \ell):=\int_{0}^{T} e^{2 i(x-T) z} \mathcal{F}(x, \ell) d x \underset{|z| \rightarrow \infty}{=} O\left(z^{2}\right) .
$$

According to (5.10) and to [22, Vol. II, Ch. 9, Section 42, Lemma 2], the entire function $\Upsilon(\cdot, \ell)$ is, in fact, a polynomial,

$$
\Upsilon(z, \ell)=a_{2}(\ell) z^{2}+a_{1}(\ell) z+a_{0}(\ell) .
$$

A comparison of the definition of $\Upsilon(z, \ell)$ in (5.10) with expression (5.11) reveals that

$$
\Upsilon(z, \ell) \equiv 0_{m_{2} \times m_{1}} .
$$

Finally, relations (5.9), (5.10), and (5.12) imply (5.1).

We recall that the right-hand side of (5.1) is continuous with respect to $x$, and so $\frac{\partial}{\partial \ell} \mathcal{A}(x, \ell)$ is continuous with respect to $x$ as well. Hence, since the matrix functions $\mathcal{A}_{k}(x, \ell)$ are continuous in $(x, \ell)$, the functions $f_{k}(\alpha)$ given by (A.40) are continuous, the equality

$$
\frac{\partial}{\partial \ell} \mathcal{A}(x, \ell)=\sum_{k=0}^{\infty} \frac{\partial}{\partial \ell} \mathcal{A}_{k}(x, \ell)
$$

is valid, equalities (A.68) and (A.79) hold, and $\widehat{\omega}(\alpha, \eta)$ in (A.79) is continuous with respect to $\alpha$ (see Remark A.10), one concludes that the matrix function $\frac{\partial}{\partial \ell} \mathcal{A}(x, \ell)$ is continuous with respect to the pair $x$ and $\ell$. Now, (5.1) yields that $\frac{\partial}{\partial x} \mathcal{A}(x, \ell)$ is also continuous with respect to $(x, \ell)$.

It remains to remove the additional condition $\operatorname{supp}(v) \subseteq[0, a]$. Indeed, according to (3.11), (4.32), and Remark 3.4. the function $\mathcal{A}(x, \ell)$, where $x \leq b_{1}$ and $\ell \leq b_{2}$, is uniquely determined by $v(x)$ for $x \in\left[0, b_{1}+b_{2}\right]$. Thus, one can indeed abandon the requirement $\operatorname{supp}(v) \subseteq[0, a]$ for (5.1) to hold. 


\section{The Inverse Approach}

Similarly to the procedure for scalar Schrödinger operators in [14, 24, 31, one can solve the inverse problem for the Dirac-type system by solving the $\mathcal{A}$-equation (5.1) with the boundary condition $\mathcal{A}(x, 0)=\mathcal{A}(x)$ (which is easily recovered from the Weyl-Titchmarsh function by taking the inverse Fourier transform in (3.14)) and by using the equality $v(\ell)=-i \mathcal{A}(0, \ell)^{*}$ in (4.33). In order to demonstrate that this approach works, we next prove the existence and uniqueness results for (5.1).

First, we consider the necessary conditions on $\mathcal{A}(x, 0)$, required in our inverse problem. It is immediate from (3.6) that for $x \in(0, T)$ one has

$$
(K f)(x)-\left(K^{*} f\right)(x)=i \gamma(x) J \int_{0}^{T} \gamma(t)^{*} f(t) d t, \quad f \in\left[L^{2}((0, T))\right]^{m_{2}} .
$$

Multiplying this equality by $E^{-1}$ from the left and by $\left(E^{-1}\right)^{*}$ from the right and taking into account (3.7), (3.9) and (3.11), one obtains the operator identity,

$$
\begin{aligned}
& \mathcal{I} S-S \mathcal{I}^{*}=i \Pi J \Pi^{*} ; \quad S:=E^{-1}\left(E^{-1}\right)^{*}, \\
& \Pi \in \mathcal{B}\left(\mathbb{C}^{m},\left[L^{2}((0, T))\right]^{m_{2}}\right), \\
& (\Pi g)(x)=\left(E^{-1} \gamma\right)(x) g=\left[\Phi(x) \quad I_{m_{2}}\right] g, \quad g \in \mathbb{C}^{m} .
\end{aligned}
$$

According to [28, Proposition 2.41], there is a unique bounded operator $S$, which satisfies the identity $A S-S A^{*}=i \Pi J \Pi^{*}$ in (6.2). This $S$ is strictly positive definite, $S>0$ (i.e., there exists $\varepsilon>0$ such that $S \geq \varepsilon I_{\left[L^{2}((0, T))\right]^{m_{2}}}$ ) and its integral kernel is expressed via $\mathcal{A}(\cdot)$. More precisely, one has

$$
\begin{aligned}
& (S f)(x)=\left(S_{T} f\right)(x)=f(x)-\int_{0}^{T} s(x, t) f(t) d t>0, \quad f \in\left[L^{2}((0, T))\right]^{m_{2}}, \\
& s(x, t):=\int_{0}^{\min (x, t)} \mathcal{A}(x-\xi) \mathcal{A}(t-\xi)^{*} d \xi \quad((x, t) \in(0, T) \times(0, T)),
\end{aligned}
$$

with

$$
S:=S_{T}>0 \quad(\text { for all } T>0) .
$$

Hence, the following corollary of Proposition 4.1 holds:

Corollary 6.1. Suppose that $v \in\left[C^{1}([0, \infty))\right]^{m_{1} \times m_{2}}$ in the Dirac-type system (1.6). Then, $\mathcal{A}=\Phi^{\prime} \in\left[C^{1}([0, \infty))\right]^{m_{2} \times m_{1}}$ and the positive definiteness condition (6.5) holds for all $T \in[0, \infty)$, that is, $S=S_{T}>0$ for all $T>0$.

A fundamental fact is that the converse statement is also valid. To prove it we need to introduce the operators $S_{x}$ in $\left[L^{2}((0, x))\right]^{m_{2}}(x \in(0, T))$ in the same way as we introduce $S_{T}$ :

$$
\left(S_{x} f\right)(y):=f(y)-\int_{0}^{x} s(y, t) f(t) d t, \quad S_{x} \in \mathcal{B}\left(\left[L^{2}((0, x))\right]^{m_{2}}\right),
$$

where $s(x, t)$ is given by the last equality in (6.4).

Proposition 6.2. Let the $m_{2} \times m_{1}$ matrix function $\mathcal{A} \in\left[C^{1}([0, \infty))\right]^{m_{2} \times m_{1}}$ satisfy the positive definiteness condition (6.5) for all $T \in(0, \infty)$. Then there exists $v \in$ $\left[C^{1}([0, \infty))\right]^{m_{1} \times m_{2}}$ in the Dirac-type system (1.6) such that $\mathcal{A}=\Phi^{\prime}$. In particular,

$$
v(\ell)=-i \mathcal{A}(0, \ell)^{*} \quad(\ell \geq 0) \text {. }
$$


Proof. It follows from the proofs of Theorem 2.54 and Lemma 2.55 in 28 that the potential $v$ in the Dirac-type system (1.6), such that one has $\Phi^{\prime}=\mathcal{A}$ (for the $m_{2} \times m_{1}$ matrix-valued function $\Phi$ corresponding to this Dirac-type system), is given by the formula $v=i \beta_{\Phi}^{\prime} J \gamma_{\Phi}$, where

$$
\begin{aligned}
& \Phi(x)=\int_{0}^{x} \mathcal{A}(t) d t, \\
& \beta_{\Phi}(x):=\left[\begin{array}{ll}
I_{m_{1}} & 0_{m_{1} \times m_{2}}
\end{array}\right]+\int_{0}^{x}\left(S_{x}^{-1} \mathcal{A}\right)(t)^{*}\left[\begin{array}{ll}
\Phi(t) & I_{m_{2}}
\end{array}\right] d t, \\
& \gamma_{\Phi}(x):=E_{\Phi}\left[\Phi(x) \quad I_{m_{2}}\right] .
\end{aligned}
$$

Here, the operators $E_{\Phi} \in \mathcal{B}\left(\left[L^{2}((0, T))\right]^{m_{2}}\right)$ are of the form

$$
\left(E_{\Phi} f\right)(x)=f(x)+\int_{0}^{x} E_{\Phi}(x, t) f(t) d t, \quad f \in\left[L^{2}((0, T))\right]^{m_{2}},
$$

with continuous integral kernels $E_{\Phi}(\cdot, \cdot)$, and they are uniquely determined by the factorizations $S_{T}^{-1}=E_{\Phi}^{*} E_{\Phi}$, where $S_{T}$ are given by (6.4). The operators $E_{\Phi}$ are applied (in (6.10) ) to the matrix function $\left[\Phi(x) \quad I_{m_{2}}\right]$ columnwise. We note that $E_{\Phi}(x, t)$ does not depend on the choice of $T$ as long as $T \geq x$.

It remains to show that $v$ is continuously differentiable (since [28, Theorem 2.54] deals with the case of locally bounded $\mathcal{A}$ and $v$ ). For that purpose, it suffices to show that $\gamma$ is continuously differentiable, that $\beta$ is two times continuously differentiable, and use $v=i \beta_{\Phi}^{\prime} J \gamma_{\Phi}$. Since $E_{\Phi}$ does not depend on $T$, one can factorize $S_{x}^{-1}$ and rewrite (6.9) in the form

$$
\beta_{\Phi}(x):=\left[\begin{array}{ll}
I_{m_{1}} & 0_{m_{1} \times m_{2}}
\end{array}\right]+\int_{0}^{x}\left(E_{\Phi} \mathcal{A}\right)(t)^{*}\left(E_{\Phi}\left[\Phi(t) \quad I_{m_{2}}\right]\right) d t .
$$

In view of (6.10) and (6.12), it suffices to prove that $E_{\Phi}$ maps continuously differentiable functions into continuously differentiable functions. The last property of $E_{\Phi}$ follows from the fact that $E_{\Phi}(x, t)$ is differentiable with respect to $x$ and both functions $E_{\Phi}(x, t)$ and $\frac{\partial}{\partial x} E_{\Phi}(x, t)(x \geq t)$ are continuous in $(x, t)$. This fact will be proven below using some results in [15, pp. 185-186].

The unique factorization $S_{T}^{-1}=E_{\Phi}^{*} E_{\Phi}$ coincides with the unique factorization in [15], that is, $E_{\Phi}(x, t)$ coincides with $V_{-}(x, t)$ in the notation of [15]. Hence, according to formula (7.9) in [15, p. 186] one has

$$
E_{\Phi}(\xi, t)=\Gamma_{\xi}(\xi, t) \quad(\xi \geq t)
$$

where $\Gamma_{\xi}(x, t)$ is the integral kernel of the operator $S_{\xi}^{-1}$,

$$
\left(S_{\xi}^{-1} f\right)(x)=f(x)+\int_{0}^{\xi} \Gamma_{\xi}(x, t) f(t) d t, \quad f \in\left[L^{2}((0, \xi))\right]^{m_{2}}
$$

(for $\xi \in(0, T)$ ). According to [15, pp. 185-186], $\Gamma_{\xi}(x, t)$ is continuous with respect to $(\xi, x, t)$ and formula $(7.10)$ in [15] holds

$$
\frac{\partial}{\partial \xi} \Gamma_{\xi}(x, t)=\Gamma_{\xi}(x, \xi) \Gamma_{\xi}(\xi, t) .
$$

The first equality in eq. (7.7) in [15] can then be rewritten in the form

$$
\Gamma_{\xi}(x, t)=s(x, t)+\int_{0}^{\xi} s(x, r) \Gamma_{\xi}(r, t) d r .
$$


In particular, one obtains

$$
\Gamma_{\xi}(\xi, t)=s(\xi, t)+\int_{0}^{\xi} s(\xi, r) \Gamma_{\xi}(r, t) d r
$$

where $s(\cdot, \cdot)$ for our case is given in (6.4). Clearly, $\frac{\partial}{\partial \xi} s(\xi, t)$ is continuous with respect to $(\xi, t), \xi \geq t$. Hence, in view of (6.15) and (6.17), $\frac{\partial}{\partial \xi} \Gamma_{\xi}(\xi, t)$ is continuous with respect to $(\xi, t), \xi \geq t$. In other words (since (6.13) is valid), $E_{\Phi}(x, t)$ is differentiable with respect to $x$ and $\frac{\partial}{\partial x} E_{\Phi}(x, t)(x \geq t)$ is continuous with respect to $(x, t)$. Hence, the potential $v$ recovered above belongs to $\left[C^{1}([0, \infty))\right]^{m_{1} \times m_{2}}$. Finally, relation (6.7) for the corresponding $\mathcal{A}$-function is clear from (4.33).

Now, we can prove the existence and uniqueness result for solutions of the $\mathcal{A}$ equation, that is, for the system (5.1). Recalling our notation

$$
\mathbb{R}_{+,+}^{2}=\left\{(x, \ell) \in \mathbb{R}^{2} \mid x \geq 0, \ell \geq 0\right\}
$$

for the first quadrant, we have the following result.

Theorem 6.3. The system (5.1) with initial condition

$$
\mathcal{A}(x, 0)=\mathcal{A}(x) \in\left[C^{1}([0, \infty))\right]^{m_{2} \times m_{1}},
$$

such that the positive definiteness property $S=S_{T}>0$ (cf. (6.5)) holds for all $T \in(0, \infty)$, has a unique solution

$$
\mathcal{A}(\cdot, \cdot) \in\left[C^{1}\left(\mathbb{R}_{+,+}^{2}\right)\right]^{m_{2} \times m_{1}} .
$$

Proof. The existence of the solution $\mathcal{A}$ is immediate after we consecutively use Proposition 6.2 and Theorem 5.1

The uniqueness is proved somewhat similar to the proof of [31, Theorem 7.1]. Indeed, set $f(r, \ell)=\mathcal{A}(r-\ell, \ell)$, then,

$$
\left(\frac{\partial}{\partial \ell} f\right)(r, \ell)=\left(\frac{\partial}{\partial \ell} \mathcal{A}\right)(r-\ell, \ell)-\left(\frac{\partial}{\partial x} \mathcal{A}\right)(r-\ell, \ell) .
$$

In view of (5.1) and (6.21), one concludes that

$$
\begin{aligned}
f\left(r, \ell_{2}\right)-f\left(r, \ell_{1}\right) & =\int_{\ell_{1}}^{\ell_{2}} \int_{0}^{r-\ell} \mathcal{A}(r-\ell-t, \ell) \mathcal{A}(0, \ell)^{*} \mathcal{A}(t, \ell) d t d \ell \\
& =\int_{\ell_{1}}^{\ell_{2}} \int_{0}^{r-\ell} f(r-t, \ell) f(\ell, \ell)^{*} f(t+\ell, \ell) d t d \ell
\end{aligned}
$$

where $r \geq \ell_{2}>\ell_{1} \geq 0$.

Next, we prove the uniqueness of the solution of (5.1), (6.19) by contradiction. Assuming that there are two solutions $\mathcal{A}_{1}$ and $\mathcal{A}_{2}$, we set $f_{k}(r, \ell)=\mathcal{A}_{k}(r-\ell, \ell)$, $k=1,2$, and use (6.22) in order to obtain

$$
\begin{aligned}
& f_{1}\left(r, \ell_{2}\right)-f_{2}\left(r, \ell_{2}\right)=f_{1}\left(r, \ell_{1}\right)-f_{2}\left(r, \ell_{1}\right) \\
& \quad+\int_{\ell_{1}}^{\ell_{2}} \int_{0}^{r-\ell}\left(\left(f_{1}(r-t, \ell)-f_{2}(r-t, \ell)\right) f_{1}(\ell, \ell)^{*} f_{1}(t+\ell, \ell)\right. \\
& \quad+f_{2}(r-t, \ell)\left(f_{1}(\ell, \ell)^{*}-f_{2}(\ell, \ell)^{*}\right) f_{1}(t+\ell, \ell) \\
& \left.\quad+f_{2}(r-t, \ell) f_{2}(\ell, \ell)^{*}\left(f_{1}(t+\ell, \ell)-f_{2}(t+\ell, \ell)\right)\right) d t d \ell .
\end{aligned}
$$


Putting

$$
F(a, \ell):=\max _{\ell \leq r \leq a}\left(\left\|f_{1}(r, \ell)-f_{2}(r, \ell)\right\|\right)
$$

(with $\|\cdot\|$ a convenient matrix norm), and taking into account (6.23), one derives

$$
F\left(a, \ell_{2}\right) \leq F\left(a, \ell_{1}\right)+\widetilde{C} \int_{\ell_{1}}^{\ell_{2}}(r-\ell) F(a, \ell) d \ell \leq F\left(a, \ell_{1}\right)+C \int_{\ell_{1}}^{\ell_{2}} F(a, \ell) d \ell
$$

for any fixed $a>0$ and some $\widetilde{C}, C>0$. Our concluding arguments now coincide with the end of the proof of [31, Theorem 7.1]: formula (6.25) yields

$$
\mathcal{F}\left(a, \ell_{2}\right) \leq \mathcal{F}\left(a, \ell_{1}\right)+C\left(\ell_{2}-\ell_{1}\right) \mathcal{F}\left(a, \ell_{2}\right),
$$

where

$$
\mathcal{F}(a, \ell):=\max _{0 \leq s \leq \ell}(F(a, s)) .
$$

According to (6.19), one concludes that $f_{k}(r, 0)=\mathcal{A}_{k}(r, 0)=\mathcal{A}(r)$, and so $F(a, 0)=$ $0_{m_{2} \times m_{1}}$. Hence, $\mathcal{F}(a, 0)=0_{m_{2} \times m_{1}}$. Therefore, formula (6.26) with $\ell_{1}=0$ (and $\ell_{2}=\ell$ ) implies that $\mathcal{F}(a, \ell)=0_{m_{2} \times m_{1}}$ for $\ell<1 / C$. Repeating this argument a finite number of times then yields $\mathcal{F}(a, \ell)=0_{m_{2} \times m_{1}}$ for all $\ell \leq a$, that is, $\mathcal{A}_{1}(x, \ell)=\mathcal{A}_{2}(x, \ell)$ in the triangle $x+\ell \leq a$. Since the equality holds for any $a>0$, the solution $\mathcal{A}(x, \ell)$ is unique.

We refer to (1.20) and (1.21) for a succinct visual summary of the direct and, especially, the inverse approach developed in this paper.

Remark 6.4. For convenience of exposition, we considered the Dirac-type system (1.6) on the half-axis and the $\mathcal{A}$-equation (5.1) on the first quadrant $\mathbb{R}_{+,+}^{2}$ (i.e., we focused on global solutions). However, our considerations are applicable to the local case as well. Indeed, given $\mathcal{A}(x, 0) \in\left[C^{1}([0, T])\right]^{m_{2} \times m_{1}}$, we use the relations $v=i \beta_{\Phi}^{\prime} J \gamma_{\Phi}$ and (6.9), 6.10) in the proof of Proposition 6.2 in order to recover the Dirac-type system on $[0, \mathrm{~T}]$ with a continuously differentiable potential $v \in$ $\left[C^{1}([0, T])\right]^{m_{1} \times m_{2}}$. Clearly, $v(\cdot)$ may be easily extended to $v \in\left[C^{1}([0, \infty))\right]^{m_{1} \times m_{2}}$. For each such extension (and associated Weyl-Titchmarsh function) the global solution of (5.1) exists, and so the local solutions exist as well. Moreover, according to the proofs of Theorem 2.54 and Lemma 2.55 in [28], the corresponding $\mathcal{A}$-function coincides on $[0, T]$ with the initial function $\mathcal{A}(x, 0)$. The uniqueness of the solution of (5.1) is also proved locally, namely, for $\mathcal{A}(x, \ell)$ in the triangle $x+\ell \leq T$ (see the proof of Theorem 6.3). To illustrate this point we briefly derive a version of the local Borg-Marchenko uniqueness theorem for Dirac-type systems (1.6) next.

Theorem 6.5. Let $\varphi(z)$ and $\widetilde{\varphi}(z)$ be the Weyl-Titchmarsh functions of two Diractype systems on the half-axis with corresponding potentials $v \in\left[C^{1}([0, \infty))\right]^{m_{1} \times m_{2}}$ and $\widetilde{v} \in\left[C^{1}([0, \infty))\right]^{m_{1} \times m_{2}}$, respectively. Suppose that on some ray $\operatorname{Re}(z)=$ $c \operatorname{Im}(z)(c \in \mathbb{R}, \operatorname{Im}(z)>0)$ for each $\varepsilon>0$ one has (again for a convenient matrix norm $\|\cdot\|)$

$$
\|\varphi(z)-\widetilde{\varphi}(z)\|_{(|z| \rightarrow \infty}^{=} O\left(e^{2 i(T-\varepsilon) z}\right)
$$

Then,

$$
v(x)=\widetilde{v}(x) \text { for all } x \in[0, T]
$$


Proof. Using (4.32), (5.2), and Proposition 4.1 one integrates in (3.13) by parts and derives

$$
\varphi(z)=-\int_{0}^{T-\varepsilon} e^{2 i x z} \mathcal{A}(x, 0) d x+O\left(z e^{2 i(T-\varepsilon) z} /(\operatorname{Im}(z))^{1 / 2}\right) \quad(T>2 \varepsilon>0) .
$$

Clearly, one has a similar formula for $\widetilde{\varphi}(z)$, implying the relation

$$
\begin{aligned}
\varphi(z)-\widetilde{\varphi}(z)= & \int_{0}^{T-2 \varepsilon} e^{2 i x z}[\widetilde{\mathcal{A}}(x, 0)-\mathcal{A}(x, 0)] d x+ \\
& +\int_{T-2 \varepsilon}^{T-\varepsilon} e^{2 i x z}[\widetilde{\mathcal{A}}(x, 0)-\mathcal{A}(x, 0)] d x+O\left(z e^{2 i(T-\varepsilon) z} /(\operatorname{Im}(z))^{1 / 2}\right) .
\end{aligned}
$$

Here, $\widetilde{\mathcal{A}}$ is the $\mathcal{A}$-function for Dirac-type system (1.6) corresponding to the potential $\widetilde{v}$. Taking into account (6.28) and (6.31), one infers that the entries of the $m_{2} \times m_{1}$ matrix-valued function

$$
W(z)=e^{-2 i(T-2 \varepsilon) z} \int_{0}^{T-2 \varepsilon} e^{2 i x z}[\widetilde{\mathcal{A}}(x, 0)-\mathcal{A}(x, 0)] d x
$$

tend to zero on the ray $\operatorname{Re}(z)=c \operatorname{Im}(z)$. According to the definition (6.32), these entries are also bounded on $\mathbb{C}_{-} \cup \mathbb{R}$ (in fact, they tend to zero). Thus, applying the Phragmen-Lindelöf theorem to the entries of $W(z)$ for the two sectors between our ray and the real line $\mathbb{R}$, one derives that $W(z)$ is constant. Moreover, since $W(z)$ tends to zero on some rays, one has $W(z) \equiv 0_{m_{2} \times m_{1}}$. Hence, $\mathcal{A}(x, 0)=\widetilde{\mathcal{A}}(x, 0)$ for all $x \in[0, T-2 \varepsilon]$ and all $\varepsilon>0$, that is, for all $x \in[0, T]$. Now, (6.29) follows from the proof of the uniqueness in Theorem 6.3 (see also Remark 6.4) and from formula 6.7).

Several versions of the local Borg-Marchenko uniqueness result, Theorem 6.5 exist in the literature under varying hypotheses on $v$. For the case of locally bounded rectangular matrices $v$ we refer to [8, 28, Section 2.3.3], for the case of locally square integrable rectangular matrics $v$, see [27]. A particular normal form of self-adjoint Dirac-type operators involving locally integrable square matrices $v$ was considered in [5]. The current proof of Theorem 6.5 based on the $\mathcal{A}$-function concept distinguishes itself due to its particular simplicity.

We emphasize once more that the results in this paper apply to matrix-valued Schrödinger operators as indicated in Remark 1.7 $(i)$.

\section{Appendix A. Various Results in Support of Step 2 In the Proof of Theorem 5.1}

We divide this appendix into three parts.

Part 1. We consider the Dirac-type system (1.6) on $[0, \infty)$, setting $u(x, z):=$ $u_{0}(x, z)$ (implying $\left.u_{0}(0, z)=I_{m}\right)$ and $\varphi(x, z):=\varphi_{0}(x, z)$ (cf. (2.2)), and introduce the differential expression $\mathcal{L}=-i J \frac{d}{d x}-V(x)$. For the fundamental solution $u(x, z)$ of the system (1.6), one has

$$
\mathcal{L} u=z u .
$$

Recalling that $\varphi$ is the Weyl-Titchmarsh function of system (1.6), it is convenient to introduce also the fundamental solutions $w(x, z)$ with a normalization at $x=0$ 
different from $u(x, z)$ :

$$
w(x, z)=u(x, z) Q(z), \quad Q(z):=\left[\begin{array}{cc}
I_{m_{1}} & 0_{m_{1} \times m_{2}} \\
\varphi(z) & I_{m_{2}}
\end{array}\right] .
$$

By $L$ we denote the operator acting in $\left[L^{2}((0, \infty))\right]^{m}$ via the differential expression $\mathcal{L}$, such that functions $Y$ in the domain of $L$ satisfy the boundary condition

$$
\left[\begin{array}{ll}
I_{m_{1}} & 0_{m_{1} \times m_{2}}
\end{array}\right] Y(0)=0_{m_{1} \times 1} .
$$

A discussion of the resolvent of Dirac-type operators on an interval can be found, for instance, in [2, Theorem 9.4.1]. In our case, the situation is similar and simple calculations show that

$$
\mathcal{L} Y_{p}=z Y_{p}+f \quad \text { for } \quad Y_{p}(x, z):=i w(x, z) \int_{0}^{x} w(t, z)^{-1} J f(t) d t,
$$

where the assumption $f \in\left[L^{2}((0, \infty))\right]^{m}$ suffices for our purposes. Formulas (A.1) and (A.4) imply that (for $g$ independent of $x \geq 0$ )

$$
\left(\mathcal{L}-z I_{\left[L^{2}((0, \infty))\right]^{m}}\right) Y(x, z)=f(x) \text { for } Y(x, z)=Y_{p}(x, z)+w(x, z) g(z) .
$$

Next, we assume that the supports of $V$ and $f$ belong to some finite interval $[0, a]$ for some $a>0$,

$$
\operatorname{supp}(V) \subseteq[0, a], \quad \operatorname{supp}(f) \subseteq[0, a] .
$$

We note that when $f, g$ and $Y$ are matrix-valued functions (instead of being vector-valued), relations like $L Y$ and $Y(\cdot, z) \in\left[L^{2}((0, \infty))\right]^{m \times r}(1 \leq r \leq m)$ are considered columnwise and the formulas above remain valid. The scalar product $\left(Y_{1}, Y_{2}\right)$ in $\left[L^{2}((0, \infty))\right]^{m}$ is understood for matrix-valued as well as vector-valued functions as

$$
\left(Y_{1}, Y_{2}\right)_{\left[L^{2}((0, \infty))\right]^{m}}=\int_{0}^{\infty} Y_{2}(x)^{*} Y_{1}(x) d x
$$

Next, we choose $g(z)$ in A.5 that the following conditions hold:

$$
Y(x, z) \in\left[L^{2}((0, \infty))\right]^{m}, \quad\left[\begin{array}{ll}
I_{m_{1}} & 0_{m_{1} \times m_{2}}
\end{array}\right] Y(0)=0_{m_{1} \times 1} .
$$

Taking into account (A.4) and (A.5), one rewrites $Y$ as follows:

$$
Y(x, z)=w(x, z)\left(g(z)+i \int_{0}^{x} w(t, z)^{-1} J f(t) d t\right) .
$$

Employing equations (A.6) and (A.8), one simplifies (A.9) for $x \geq a$ and fixes the required function $g(z)$ as follows

$$
\begin{aligned}
& Y(x, z)=w(x, z)\left(g(z)+i \int_{0}^{a} w(t, z)^{-1} J f(t) d t\right) \quad \text { for } \quad x \geq a, \\
& g(z)=\left[\begin{array}{l}
g_{1}(z) \\
g_{2}(z)
\end{array}\right], \quad g_{1}(z)=0_{m_{1}}, \quad g_{2}(z)=-i\left[\begin{array}{ll}
0_{m_{2} \times m_{1}} & I_{m_{2}}
\end{array}\right] \int_{0}^{a} w(t, z)^{-1} J f(t) d t .
\end{aligned}
$$

Indeed, formulas (A.2), (A.10), and (A.11) show that $Y(x, z)$ for $x \geq a$ takes on the form

$$
Y(x, z)=u(x, z)\left[\begin{array}{l}
I_{m_{1}} \\
\varphi(z)
\end{array}\right] c(z) .
$$

Hence, in view of (1.9), the first condition in (A.8) is fulfilled. The second relation in (A.8) follows from (A.9), from the normalization $w(0, z)=Q(z)$ and from the 
equality $g_{1}(z)=0$. It is easy to see that the requirements (A.11) are not only sufficient but also necessary for (A.8) to be valid.

Formulas (A.5) and (A.8) prove the following result.

Lemma A.1. Suppose that $f \in\left[L^{2}((0, \infty))\right]^{m}$ and $\operatorname{supp}(f) \subseteq[0, a]$. Then

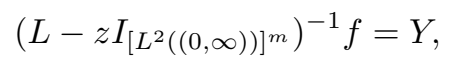

where $Y(x, z)$ is given by (A.9) and A.11).

Taking into account (1.6), one obtains the equalities

$$
\begin{aligned}
& \frac{d}{d t}\left(w(t, z)^{-1} e^{i t z J}\right)=w(t, z)^{-1}(-i) J V(t) e^{i t z J}, \\
& \frac{d}{d x}\left(e^{-i x z J} u(x, z)\right)=e^{-i x z J} i J V(x) u(x, z) .
\end{aligned}
$$

Equalities A.13) - A.15) are essential in the proof of the next proposition.

Proposition A.2. Suppose that $V$ satisfies the conditions $V \in\left[L^{2}((0, \infty))\right]^{m \times m}$ and $\operatorname{supp}(V) \subseteq[0, a]$. Then

$$
\begin{aligned}
& \varphi(z)=i \int_{0}^{a} e^{2 i x z} v(x)^{*} d x+i\left(\left(L-z I_{\left.\left[L^{2}((0, \infty))\right]^{m}\right)^{-1}} f_{1}, f_{2}\right),\right. \\
& f_{1}(x, z):=V(x) e^{i x z J}\left[\begin{array}{c}
I_{m_{1}} \\
0
\end{array}\right], \quad f_{2}:=V(x) e^{i x z J}\left[\begin{array}{c}
0 \\
I_{m_{2}}
\end{array}\right] .
\end{aligned}
$$

Proof. Taking into account (A.9), (A.13) and (A.14), one rewrites $(L-z I)^{-1} f_{1}$, where $f_{1}$ is given in (A.17), in the form

$$
\left(L-z I_{\left[L^{2}((0, \infty))\right]^{m}}\right)^{-1} f_{1}=\left(u(x, z)-e^{i x z J}\right)\left[\begin{array}{c}
I_{m_{1}} \\
0_{m_{2} \times m_{1}}
\end{array}\right]+w(x, z) g(z) .
$$

Next, in view of (A.11), (A.14), and (A.17), one derives

$$
g(z)=\left[\begin{array}{c}
0_{m_{1} \times m_{2}} \\
I_{m_{2}}
\end{array}\right]\left[\begin{array}{ll}
0_{m_{2} \times m_{1}} & I_{m_{2}}
\end{array}\right]\left(w(a, z)^{-1} e^{i a z J}-Q(z)^{-1}\right)\left[\begin{array}{c}
I_{m_{1}} \\
0_{m_{2} \times m_{1}}
\end{array}\right] .
$$

Using (A.2), one rewrites (A.19) as

$$
\begin{aligned}
g(z) & =\left[\begin{array}{c}
0_{m_{1} \times m_{2}} \\
I_{m_{2}}
\end{array}\right]\left[-\varphi(z) \quad I_{m_{2}}\right]\left(u(a, z)^{-1} e^{i a z J}-I_{m}\right)\left[\begin{array}{c}
I_{m_{1}} \\
0_{m_{2} \times m_{1}}
\end{array}\right] \\
& =\left[\begin{array}{c}
0_{m_{1} \times m_{2}} \\
I_{m_{2}}
\end{array}\right]\left(\varphi(z)+\left[\begin{array}{ll}
-\varphi(z) & I_{m_{2}}
\end{array}\right] u(a, z)^{-1} e^{i a z J}\left[\begin{array}{c}
I_{m_{1}} \\
0_{m_{2} \times m_{1}}
\end{array}\right]\right) .
\end{aligned}
$$

Since $\operatorname{supp}(V) \subseteq[0, a]$, one has $u(x, z)=e^{i(x-a) z J} u(a, z)$ for $x \geq a$. Therefore, one obtains the relation

$$
u(x, z)\left[\begin{array}{c}
I_{m_{1}} \\
\varphi(z)
\end{array}\right]=e^{i(x-a) z J} u(a, z)\left[\begin{array}{c}
I_{m_{1}} \\
\varphi(z)
\end{array}\right] \in\left[L^{2}((0, \infty))\right]^{m}
$$

implying that $\left[\begin{array}{ll}0_{m_{2} \times m_{1}} & I_{m_{2}}\end{array}\right] u(a, z)\left[\begin{array}{c}I_{m_{1}} \\ \varphi(z)\end{array}\right]=0_{m_{2} \times m_{1}}$ (in view of $\left.\operatorname{Im}(z)>0\right)$. That is,

$\varphi(z)=-u_{22}(a, z)^{-1} u_{21}(a, z), \quad\left[-\varphi(z) \quad I_{m_{2}}\right]=u_{22}(a, z)^{-1}\left[u_{21}(a, z) \quad u_{22}(a, z)\right]$. 
Formula A.20 and the second equality in A.22 yield

$$
g(z)=\left[\begin{array}{l}
0_{m_{1}} \\
\varphi(z)
\end{array}\right]
$$

Finally, from (A.18) and (A.23) one concludes that

$$
\left(L-z I_{\left[L^{2}((0, \infty))\right]^{m}}\right)^{-1} f_{1}=u(x, z)\left[\begin{array}{c}
I_{m_{1}} \\
\varphi(z)
\end{array}\right]-e^{i x z J}\left[\begin{array}{c}
I_{m_{1}} \\
0_{m_{2} \times m_{1}}
\end{array}\right] .
$$

By virtue of (A.17) and (A.24) one has

$$
\begin{aligned}
& \left(\left(L-z I_{\left[L^{2}((0, \infty))\right]^{m}}\right)^{-1} f_{1}, f_{2}\right)=\left[\begin{array}{ll}
0_{m_{2} \times m_{1}} & I_{m_{2}}
\end{array}\right] \int_{0}^{a} e^{-i x z J} V(x) u(x, z) d x\left[\begin{array}{c}
I_{m_{1}} \\
\varphi(z)
\end{array}\right] \\
& -\int_{0}^{a} e^{2 i x z} v(x)^{*} d x
\end{aligned}
$$

Employing (A.15), one simplifies (A.25) as follows,

$$
\begin{aligned}
((L- & \left.\left.z I_{\left[L^{2}((0, \infty))\right]^{m}}\right)^{-1} f_{1}, f_{2}\right)=i\left[\begin{array}{ll}
0_{m_{2} \times m_{1}} & I_{m_{2}}
\end{array}\right]\left(e^{-i a z J} u(a, z)-I_{m}\right)\left[\begin{array}{c}
I_{m_{1}} \\
\varphi(z)
\end{array}\right] \\
& -\int_{0}^{a} e^{2 i x z} v(x)^{*} d x \\
= & -i \varphi(z)+i e^{i a z}\left[u_{21}(a, z) \quad u_{22}(a, z)\right]\left[\begin{array}{c}
I_{m_{1}} \\
\varphi(z)
\end{array}\right]-\int_{0}^{a} e^{2 i x z} v(x)^{*} d x
\end{aligned}
$$

Finally, applying the first equality in (A.22) one derives

$$
\left(\left(L-z I_{\left[L^{2}((0, \infty))\right]^{m}}\right)^{-1} f_{1}, f_{2}\right)=-i \varphi(z)-\int_{0}^{a} e^{2 i x z} v(x)^{*} d x
$$

which is A.16).

Since

$$
e^{i x z J}=\left[\begin{array}{cc}
e^{i x z} I_{m_{1}} & 0_{m_{1} \times m_{2}} \\
0_{m_{2} \times m_{1}} & e^{-i x z} I_{m_{2}}
\end{array}\right]
$$

(A.17) is equivalent to

$$
f_{1}(x, z)=e^{i x z}\left[\begin{array}{c}
0_{m_{1}} \\
v(x)^{*}
\end{array}\right], \quad f_{2}=e^{-i x \bar{z}}\left[\begin{array}{c}
v(x) \\
0_{m_{2}}
\end{array}\right] .
$$

In the special case $V=0_{m \times m}$ a.e., we write $\mathcal{L}_{0}$ and $L_{0}$ instead of $\mathcal{L}$ and $L$ and note that formulas A.13) and A.9 yield

$$
\begin{aligned}
& \left(\left(L_{0}-z I_{\left[L^{2}((0, \infty))\right]^{m}}\right)^{-1} f\right)(x)=\int_{0}^{\infty} G_{0}\left(z, x, x^{\prime}\right) f\left(x^{\prime}\right) d x^{\prime}, \\
& G_{0}\left(z, x, x^{\prime}\right)=i e^{i z\left|x-x^{\prime}\right|} \begin{cases}{\left[\begin{array}{cc}
I_{m_{1}} & 0_{m_{1} \times m_{2}} \\
0_{m_{2} \times m_{1}} & 0_{m_{2}}
\end{array}\right],} & x>x^{\prime}, \\
{\left[\begin{array}{cc}
0_{m_{1}} & 0_{m_{1} \times m_{2}} \\
0_{m_{2} \times m_{1}} & I_{m_{2}}
\end{array}\right],} & x<x^{\prime},\end{cases}
\end{aligned}
$$


Moreover, according to (A.31), the Neumann series

$$
\left(L-z I_{\left[L^{2}((0, \infty))\right]^{m}}\right)^{-1}=\sum_{n=0}^{\infty}\left(L_{0}-z I_{\left[L^{2}((0, \infty))\right]^{m}}\right)^{-1}\left[V\left(L_{0}-z I_{\left[L^{2}((0, \infty))\right]^{m}}\right)^{-1}\right]^{n}
$$

is norm convergent on the subspace $\left[L^{2}((0, a))\right]^{m}$ for $0<\operatorname{Im}(z)$ sufficiently large, and we now employ it to shed additional light on the Weyl-Titchmarsh function $\varphi$ with the help of (A.16).

Lemma A.3. Suppose that $V$ satisfies $V \in\left[L^{2}((0, \infty))\right]^{m \times m}$ and $\operatorname{supp}(V) \subseteq[0, a]$. Then for $0<\operatorname{Im}(z)$ sufficiently large,

$$
\begin{aligned}
\varphi(z) & =i \int_{0}^{a} e^{2 i x z} v(x)^{*} d x+i\left(\left(L-z I_{\left[L^{2}((0, \infty))\right]^{m}}\right)^{-1} f_{1}, f_{2}\right) \\
& =-\sum_{k=0}^{\infty} M_{2 k+1}(z ; V),
\end{aligned}
$$

where

$$
\begin{aligned}
M_{1}(z ; V)= & -i \int_{0}^{a} e^{2 i z x_{0}} v\left(x_{0}\right)^{*} d x_{0}:=\int_{0}^{a} e^{2 i z \alpha} \mathcal{A}_{1}(\alpha) d \alpha \\
M_{2 k+1}(z ; V)= & (-1)^{k+1} i \int_{0}^{a} d x_{2 k} \int_{0}^{x_{2 k}} d x_{2 k-1} \int_{x_{2 k-1}}^{a} d x_{2 k-2} \cdots \int_{0}^{x_{2}} d x_{1} \int_{x_{1}}^{a} d x_{0} \\
& \times e^{2 i z \alpha} v\left(x_{2 k}\right)^{*} v\left(x_{2 k-1}\right) v\left(x_{2 k-2}\right)^{*} \cdots v\left(x_{1}\right) v\left(x_{0}\right)^{*} \\
\alpha= & x_{2 k}-x_{2 k-1}+x_{2 k-2}-\ldots-x_{1}+x_{0} .
\end{aligned}
$$

Proof. Insertion of the Neumann series (A.32) into formula (A.16) for $\varphi$, exploiting the explicit form of $G_{0}(z, \cdot, \cdot)$ in (A.31), yields (A.33) and (A.34). In particular, the special block matrix structure of $V$ and $\left(L_{0}-z I\right)^{-1}$, and the form of $f_{j}, j=1,2$, shows that all even terms vanish identically in the Neumann expansion inserted into the first line of (A.33),

$$
\left(\left(L_{0}-z I_{\left[L^{2}((0, \infty))\right]^{m}}\right)^{-1}\left[V\left(L_{0}-z I_{\left[L^{2}((0, \infty))\right]^{m}}\right)^{-1}\right]^{2 k} f_{1}, f_{2}\right)=0, \quad k \in \mathbb{N}_{0} .
$$

In addition, we introduce the notation

$$
\begin{aligned}
& \ell_{0}=\alpha=x_{2 k}-x_{2 k-1}+x_{2 k-2} \ldots+x_{2}-x_{1}+x_{0}, \\
& \ell_{1}=x_{2 k}-x_{2 k-1}+x_{2 k-2} \ldots+x_{2}-x_{1}, \\
& \ell_{2}=x_{2 k}-x_{2 k-1}+x_{2 k-2} \ldots+x_{2}, \quad \ldots
\end{aligned}
$$

Part 2. Changing the order of integration in (A.34) one may derive the following lemma.

Lemma A.4. Suppose that $V$ satisfies $V \in\left[L^{2}((0, \infty))\right]^{m \times m}$ and $\operatorname{supp}(V) \subseteq[0, a]$. Then $M_{2 k+1}$ admits representation

$$
M_{2 k+1}(z ; V)=\int_{0}^{(k+1) a} e^{2 i z \alpha} \mathcal{A}_{k}(\alpha) d \alpha .
$$

Moreover, for continuous $v$ such that

$$
\max \|v(x)\| \leq c \quad(c \in(0, \infty))
$$


one obtains

$$
\left\|\mathcal{A}_{k}(\alpha)\right\| \leq c^{2 k+1} f_{k}(\alpha)
$$

where $f_{k}(\alpha)$ (which does not depend on $c$ ) is given by

$$
f_{k}(\alpha)=(-1)^{k+1} \widehat{\mathcal{A}}_{k}(\alpha),
$$

and $\widehat{\mathcal{A}}_{k}(\alpha)$ is $\mathcal{A}_{k}(\alpha)$ for the special case

$$
v(x)=\widehat{v}(x):= \begin{cases}i, & x \in[0, a], \\ 0, & x \in(a, \infty) .\end{cases}
$$

Proof. Recalling (A.36) and putting $x_{0}=\alpha-\ell_{1}$, one gets

$$
\begin{aligned}
\Lambda\left(\alpha-\ell_{1}, x_{1}, \ldots, x_{2 k}\right) & =\Lambda\left(x_{0}, x_{1}, \ldots, x_{2 k}\right) \\
& =i v\left(x_{2 k}\right)^{*} v\left(x_{2 k-1}\right) v\left(x_{2 k-2}\right)^{*} \cdots v\left(x_{1}\right) v\left(x_{0}\right)^{*} .
\end{aligned}
$$

Next, changing variables and the order of integration in (A.34) one obtains

$$
\begin{aligned}
& \int_{0}^{x_{2}} d x_{1} \int_{x_{1}}^{a} d x_{0} e^{2 i z \alpha} \Lambda\left(x_{0}, x_{1}, \ldots, x_{2 k}\right) \\
& =\int_{\ell_{2}}^{a+\ell_{3}} d \alpha \int_{0}^{x_{2}} d x_{1} e^{2 i z \alpha} \Lambda\left(\alpha-\ell_{1}, x_{1}, \ldots, x_{2 k}\right) \\
& \quad+\int_{a+\ell_{3}}^{a+\ell_{2}} d \alpha \int_{0}^{a+\ell_{2}-\alpha} d x_{1} e^{2 i z \alpha} \Lambda\left(\alpha-\ell_{1}, x_{1}, \ldots, x_{2 k}\right) .
\end{aligned}
$$

One can see by induction that the change of order of integration with respect to $\alpha$ and with respect to $x_{2 s}, x_{2 s+1}$ generates integrals of the form $\int_{(r-1) a+\ell_{2 s+2}}^{r a+\ell_{2 s+3}} d \alpha$ or $\int_{r a+\ell_{2 s+3}}^{r a+\ell_{2 s+2}} d \alpha$, which follows from (A.43) and the next two equalities:

$$
\begin{aligned}
& \int_{0}^{x_{2 s+2}} d x_{2 s+1} \int_{x_{2 s+1}}^{a} d x_{2 s} \int_{(r-1) a+\ell_{2 s}}^{r a+\ell_{2 s+1}} d \alpha \\
& =\int_{(r-1) a+\ell_{2 s+2}}^{r a+\ell_{2 s+3}} d \alpha \int_{0}^{x_{2 s+2}} d x_{2 s+1} \int_{x_{2 s+1}}^{\alpha-(r-1) a-\ell_{2 s+1}} d x_{2 s} \\
& \quad+\int_{r a+\ell_{2 s+3}}^{r a+\ell_{2 s+2}} d \alpha \int_{0}^{r a+\ell_{2 s+2}-\alpha} d x_{2 s+1}^{\alpha-(r-1) a-\ell_{2 s+1}} d x_{x_{2 s+1}}^{\alpha}
\end{aligned}
$$

and

$$
\begin{aligned}
& \int_{0}^{x_{2 s+2}} d x_{2 s+1} \int_{x_{2 s+1}}^{a} d x_{2 s} \int_{r a+\ell_{2 s+1}}^{r a+\ell_{2 s}} d \alpha \\
& =\int_{r a+\ell_{2 s+3}}^{r a+\ell_{2 s+2}} d \alpha \int_{r a+\ell_{2 s+2}-\alpha}^{x_{2 s+2}} d x_{2 s+1} \int_{x_{2 s+1}}^{a} d x_{2 s} \\
& \quad+\int_{r a+\ell_{2 s+2}}^{(r+1) a+\ell_{2 s+3}} d \alpha \int_{0}^{x_{2 s+2}} d x_{2 s+1} \int_{\alpha-r a-\ell_{2 s+1}}^{a} d x_{2 s} \\
& \quad+\int_{(r+1) a+\ell_{2 s+3}}^{(r+1) a+\ell_{2 s+2}} d \alpha \int_{0}^{(r+1) a+\ell_{2 s+2}-\alpha} d x_{2 s+1}^{a} \int_{\alpha-r a-\ell_{2 s+1}}^{a} d x_{2 s} .
\end{aligned}
$$


The last changes of order of integration in (A.34) are given by one of the equalities

$$
\begin{aligned}
& \int_{0}^{a} d x_{2 k} \int_{r a}^{r a+x_{2 k}} d \alpha=\int_{r a}^{(r+1) a} d \alpha \int_{\alpha-r a}^{a} d x_{2 k} \quad(r \leq k), \\
& \int_{0}^{a} d x_{2 k} \int_{(r-1) a+x_{2 k}}^{r a} d \alpha=\int_{(r-1) a}^{r a} d \alpha \int_{0}^{\alpha-(r-1) a} d x_{2 k} \quad(r \leq k) .
\end{aligned}
$$

The representation (A.37) follows from (A.34) and from A.43 A.47). Taking also equations (A.38) and (A.42) into account, one derives (A.39).

Remark A.5. We note that since $\|v(x)\|$ is bounded (i.e., (A.38) holds), we use in our estimates for $\mathcal{A}_{k}$ and in the proof of the convergence of the corresponding series for $\mathcal{A}$ several results on Dirac-type system with the special scalar $v=\widehat{v}$ given by (A.41). These results provide the necessary estimates on the iterated integrals, which appear in our calculations, and we resorted to this trick so the matrix structure in all quantities involved does not unnecessarily obscure the essential estimates.

For our estimates we need the Weyl-Titchmarsh function $\widetilde{\varphi}(z, c)$ of the system (1.6) with $v=c \widehat{v}$, where $\widehat{v}$ is given by (A.41). In the following lemma we abandon the requirement $c>0$ and consider $c \in \mathbb{C}, c \neq 0$ because the proof in this more general case remains the same and the example is of a certain independent interest.

Lemma A.6. Let $c \in \mathbb{C}, c \neq 0$, and consider the special case where

$$
v(x)=\widetilde{v}(x):=c \widehat{v}(x)= \begin{cases}c i, & x \in[0, a], \\ 0, & x \in(a, \infty),\end{cases}
$$

in the system (1.6) on $[0, \infty)$. Then the associated Weyl-Titchmarsh function $\widetilde{\varphi}(z, c)$ has the form

$$
\begin{aligned}
& \widetilde{\varphi}(z, c)=\left.\psi(z, \lambda)\right|_{\lambda=|c|^{2}}, \quad \psi(z, \lambda)=i \bar{c} \frac{1-e^{2 i q(z, \lambda)}}{z+q(z, \lambda)-(z-q(z, \lambda)) e^{2 i q(z, \lambda)}}, \\
& q(z, \lambda):=\left(z^{2}-\lambda\right)^{1 / 2} \quad\left(\operatorname{Im}(z)>0, \operatorname{Im}\left(\left(z^{2}-\lambda\right)^{1 / 2}\right)>0\right) .
\end{aligned}
$$

Proof. Introducing the $2 \times 2$ matrix-valued functions

$$
D(z)=\operatorname{diag}\left\{q\left(z,|c|^{2}\right),-q\left(z,|c|^{2}\right)\right\}, \quad K(z):=\left[\begin{array}{cc}
-i c & -i c \\
z-q\left(z,|c|^{2}\right) & z+q\left(z,|c|^{2}\right)
\end{array}\right],
$$

a direct calculation shows that

$$
K(z) D(z) K(z)^{-1}=\left[\begin{array}{cc}
z & i c \\
i \bar{c} & -z
\end{array}\right] .
$$

It follows that in the case of $v=\widetilde{v}$ given by (A.48) the fundamental solution $u(x, z)$ of (1.6) is given by the formula

$$
u(x, z)=\widetilde{u}(x, z):= \begin{cases}K(z) e^{i x D(z)} K(z)^{-1}, & x \leq a, \\ e^{i(x-a) z J} K(z) e^{i a D(z)} K(z)^{-1}, & x>a .\end{cases}
$$

In view of A.53) and the inequality $\operatorname{Im}(z)>0$, one concludes that the relation

$$
\left(K(z) e^{i a D(z)} K(z)^{-1}\right)_{21}+\left(K(z) e^{i a D(z)} K(z)^{-1}\right)_{22} \widetilde{\varphi}(z, c)=0
$$


(for the lower entries of $K(z) e^{i a D(z)} K(z)^{-1}$ ) yields the property

$$
\widetilde{u}(\cdot, z)\left[\begin{array}{c}
1 \\
\widetilde{\varphi}(z, c)
\end{array}\right] \in\left[L^{2}([0, \infty))\right]^{2}
$$

which is characteristic for the Weyl-Titchmarsh function (cf. (1.9)). In other words, one has

$$
\widetilde{\varphi}(z, c)=-\left(K(z) e^{i a D(z)} K(z)^{-1}\right)_{21} /\left(K(z) e^{i a D(z)} K(z)^{-1}\right)_{22} .
$$

Explicit calculations then show that (A.56) is equivalent to (A.49).

In the following we return to the special case $c>0$. Assuming, for instance, that $\operatorname{Im}(z) \geq 2^{1 / 2} c$, one verifies that $q(z, \lambda)$ is well-defined by A.50 and is analytic with respect to $\lambda$ in the disk $|\lambda| \leq|c|^{2}+\varepsilon$ for some $\varepsilon>0$, which does not depend on $z$. Next, one rewrites the denominator in A.49) in the form

$$
z+q(z, \lambda)-(z-q(z, \lambda)) e^{2 i q(z, \lambda)}=(z+q(z, \lambda))\left(1-\frac{\lambda}{(z+q(z, \lambda))^{2}} e^{2 i q(z, \lambda)}\right)
$$

Since $\operatorname{Im}(z) \geq 2^{1 / 2} c$, this implies $\left|(z+q(z, \lambda))^{2}\right|>2|c|^{2}$. Hence, according to (A.57), the denominator of $\psi$ does not vanish in the disk $|\lambda| \leq|c|^{2}+\varepsilon$, and $\psi$ is analytic with respect to $\lambda$ in this disk. Therefore, $\psi(z, \lambda)$ admits a Taylor expansion, which (taking into account (A.49) we compare with the expansion (A.33), (A.34) for $\widetilde{\varphi}$. It follows that

$$
\left.\frac{1}{k !} \frac{\partial^{k} \psi(z, \lambda)}{\partial \lambda^{k}}\right|_{\lambda=0} c^{2 k}=-\widetilde{M}_{2 k+1}(z, c),
$$

where $\widetilde{M}_{2 k+1}(z, c)$ is $M_{2 k+1}(z, V)$ with $v=\widetilde{v}$ given by (A.48). In view of (A.37), (A.40) and (A.58) one derives

$$
\left.\frac{1}{c} \frac{(-1)^{k}}{k !} \frac{\partial^{k} \psi(z, \lambda)}{\partial \lambda^{k}}\right|_{\lambda=0}=\int_{0}^{(k+1) a} e^{2 i z \alpha} f_{k}(\alpha) d \alpha
$$

Since $\psi(z, \lambda)$ admits Taylor expansion in the disk $|\lambda| \leq|c|^{2}+\varepsilon$, one concludes that $\left.\sum_{k=0}^{\infty} \frac{1}{c} \frac{(-1)^{k}}{k !} \frac{\partial^{k} \psi(z, \lambda)}{\partial \lambda^{k}}\right|_{\lambda=0} c^{2 k}$ converges absolutely, and, moreover,

$$
\left.\sum_{k=0}^{\infty} \frac{1}{c} \frac{(-1)^{k}}{k !} \frac{\partial^{k} \psi(z, \lambda)}{\partial \lambda^{k}}\right|_{\lambda=0} c^{2 k}=\psi\left(z,-c^{2}\right) / c
$$

Relations A.49 and A.57) imply also that $\psi\left(\xi+i \eta,-c^{2}\right) / c \in L^{2}(\mathbb{R} ; d \xi)$ (slightly abusing notation for the sake of simplicity) for all sufficiently large fixed values of $\eta$.

Remark A.7. We recall that in the case of bounded $v$ (more precisely in the case where (A.38) is valid) formula A.33 holds for $\operatorname{Im}(z) \geq \frac{1}{2} a c^{2}$ and that in the considerations after the proof of Lemma A.6 we assumed that $\operatorname{Im}(z) \geq 2^{1 / 2} c$. Thus, (A.33) and the statements and relations after the proof of Lemma A.6 hold if

$$
\eta=\operatorname{Im}(z) \geq C=\max \left(2^{1 / 2} c, a c^{2} / 2\right) .
$$


When (A.61) is valid, it follows from (A.59) and A.60 that

$$
\psi\left(\xi+i \eta,-c^{2}\right) / c=\lim _{N \rightarrow \infty} \int_{0}^{(N+1) a} e^{2 i z \alpha}\left(\sum_{k=0}^{N} f_{k}(\alpha) c^{2 k}\right) d \alpha .
$$

Formula (A.62) yields that the function $\psi\left(\xi+i \eta,-c^{2}\right) / c$ is positive definite (as a function of $\xi$ ) for each fixed $\eta$ satisfying (A.61). Furthermore, according to the properties of the positive definite functions (see, e.g., 20] and more references in 11]) the derivative $\omega(\alpha, \eta)$ of the absolutely continuous part of the distribution for the positive definite function $\psi\left(\xi+i \eta,-c^{2}\right) / c$ satisfies the following relations

$$
\omega(\alpha, \eta) \in L^{1}(\mathbb{R} ; d \alpha), \quad 2 \omega(\alpha, \eta) \geq e^{-\eta \alpha} \sum_{k=0}^{\infty} c^{2 k} f_{k}(\alpha / 2) \quad(\alpha \geq 0) .
$$

Using (A.39) and A.63), one concludes that $\sum_{k=0}^{\infty} \mathcal{A}_{k}(\alpha)$ converges on each finite interval (in the $L^{1}$-norm) and that

$$
e^{-2 \eta \alpha} \sum_{k=0}^{\infty} \mathcal{A}_{k}(\alpha) \in\left[L^{1}((0, \infty) ; d \alpha)\right]^{m_{2} \times m_{1}} .
$$

Since relations (A.33) and (A.37) imply

$$
\mathcal{A}(\alpha)=\sum_{k=0}^{\infty} \mathcal{A}_{k}(\alpha)
$$

one finally concludes that

$$
e^{-2 \eta \alpha} \mathcal{A}(\alpha) \in\left[L^{1}((0, \infty) ; d \alpha)\right]^{m_{2} \times m_{1}} .
$$

Part 3. Next, we consider in greater detail the case $v \in\left[C^{1}([0, \infty))\right]^{m_{1} \times m_{2}}$ (as in Theorem 5.1), $\operatorname{supp}(v) \subseteq[0, a]$. Then, for each $v$ there are some values $c, \widehat{c}$ such that

$$
\max (\|v(x)\|)<c, \quad \max \left(\left\|v^{\prime}(x)\right\|\right)<\widehat{c} .
$$

One recalls that we switch from $\varphi(z)$ and $\mathcal{A}(\alpha)$ to $\varphi_{\ell}(z)$ and $\mathcal{A}(x, \ell)$ by considering $v(x+\ell)$ instead of $v(x)$. Hence, (A.34), A.37) and (A.67) imply that

$$
\left\|\frac{\partial}{\partial \ell} \mathcal{A}_{k}(\alpha, \ell)\right\| \leq(2 k+1) \widehat{c} c^{2 k} f_{k}(\alpha)
$$

(cf. A.39) ). On the other hand, similarly to A.60), one derives

$$
\left.\sum_{k=1}^{\infty} \frac{(-1)^{k}}{(k-1) !} \frac{\partial^{k} \psi(z, \lambda)}{c \partial \lambda^{k}}\right|_{\lambda=0} c^{2 k-2}=-\left.\frac{1}{c} \frac{\partial \psi(z, \lambda)}{\partial \lambda}\right|_{\lambda=-c^{2}},
$$

and the series in A.69) converges absolutely. Taking into account A.59 and (A.69) one can see that the function $-\left.\frac{1}{c} \frac{\partial \psi(z, \lambda)}{\partial \lambda}\right|_{\lambda=-c^{2}}$ is positive definite. Moreover, according to the properties of the positive definite functions [20] and because of relations (A.59) and (A.69) one has (similarly to (A.63)

$$
\widehat{\omega}(\alpha, \eta) \in L^{1}(\mathbb{R} ; d \alpha), \quad 2 \widehat{\omega}(\alpha, \eta) \geq e^{-\eta \alpha} \sum_{k=1}^{\infty} k c^{2 k} f_{k}(\alpha / 2),
$$


where $\widehat{\omega}(\alpha, \eta)$ is the derivative of the absolutely continuous part of the distribution for the function $-\left.\frac{1}{c} \frac{\partial \psi(z, \lambda)}{\partial \lambda}\right|_{\lambda=-c^{2}}$. This implies that the sum

$$
\sum_{k=0}^{\infty}(2 k+1) \widehat{c} c^{2 k} f_{k}(\alpha)
$$

converges in $L^{1}((0, \infty) ; d \alpha)$ and that

$$
e^{-\eta \alpha} \sum_{k=0}^{\infty}(2 k+1) \widehat{c} c^{2 k} f_{k}(\alpha / 2) \in L^{1}((0, \infty) ; d \alpha) \quad(\eta \geq C) .
$$

Finally, by virtue of (A.65), A.68) and (A.72), one infers that $\mathcal{A}(\alpha, \ell)$ is differentiable with respect to $\ell$ and

$$
e^{-\eta \alpha} \frac{\partial}{\partial \ell} \mathcal{A}(\alpha, \ell) \in L^{1}((0, \infty) ; d \alpha)^{m_{2} \times m_{1}} \quad(\eta \geq 2 C) .
$$

Hence, relations (3.14), (4.32) and (5.2) yield the following lemma.

Lemma A.8. Suppose that $v$ satisfies $v \in\left[C^{1}([0, \infty))\right]^{m_{1} \times m_{2}}$ and $\operatorname{supp}(v) \subseteq[0, a]$. Then, the equality

$$
\frac{d}{d \ell} \varphi_{\ell}(z)=-\int_{0}^{\infty} e^{2 i \alpha z} \frac{\partial}{\partial \ell} \mathcal{A}(\alpha, \ell) d \alpha
$$

is valid for $\operatorname{Im}(z) \geq C$ (cf. (A.61) $)$.

According to (A.73) and A.74 one has the following result.

Corollary A.9. Suppose that $v$ satisfies $v \in\left[C^{1}([0, \infty))\right]^{m_{1} \times m_{2}}$ and $\operatorname{supp}(v) \subseteq$ $[0, a]$. Then

$$
\frac{d}{d \ell} \varphi_{\ell}(z) \underset{\operatorname{Im}(z) \rightarrow \infty}{=}-\int_{0}^{T} e^{2 i x z} \frac{\partial}{\partial \ell} \mathcal{A}(x, \ell) d x+O\left(e^{2 i T z}\right) .
$$

Finally, we study $-\left.\frac{1}{c} \frac{\partial \psi(z, \lambda)}{\partial \lambda}\right|_{\lambda=-c^{2}}$ in greater detail. Explicit calculations show that

$$
\frac{\partial \psi(z, \lambda)}{c \partial \lambda}=\frac{i}{2} \frac{1+4 i a q(z, \lambda) e^{2 i a q(z, \lambda)}-e^{4 i a q(z, \lambda)}}{q(z, \lambda)(z+q(z, \lambda))^{2}\left(1-\frac{\lambda}{(z+q(z, \lambda))^{2}} e^{2 i q(z, \lambda)}\right)^{2}},
$$

and so in view of (A.50) and (A.76) one has

$$
-\left.\frac{\partial \psi(\xi+i \eta, \lambda)}{c \partial \lambda}\right|_{\lambda=-c^{2}} \in L^{1}(\mathbb{R} ; d \xi) \quad(\eta \geq C) .
$$

Remark A.10. According to [30, Theorem 1.3.6], A.77) implies that the distribution functions $\mu(\alpha, \eta)$ are absolutely continuous and the densities

$$
\frac{d}{d \alpha} \mu(\alpha, \eta):=\widehat{\omega}(\alpha, \eta)
$$

are continuous (with respect to $\alpha$ ). In particular, one infers that inequality (A.70) is, in fact, an equality:

$$
2 \widehat{\omega}(\alpha, \eta)=e^{-\eta \alpha} \sum_{k=1}^{\infty} k c^{2 k} f_{k}(\alpha / 2) \quad(\eta \geq C) .
$$


Acknowledgments. We are indebted to Mark Ashbaugh for helpful discussions on the material in Part 2 of Appendix A. F.G. is indebted to Alexander Sakhnovich for the great hospitality extended to him at the Faculty of Mathematics of the University of Vienna, Austria, during an extended stay in June of 2018. The research of A.L. Sakhnovich was supported by the Austrian Science Fund (FWF) under Grant No. P29177.

\section{REFERENCES}

[1] R. A. Adams and J. J. F. Fournier, Sobolev Spaces, 2nd ed., Elsevier, San Diego, 2003.

[2] F. V. Atkinson, Discrete and Continuous Boundary Problems, Academic Press, New York, 1964.

[3] I. M. Gel'fand and B. M. Levitan, On the determination of a differential equation from its special function, Izv. Akad. Nauk SSR. Ser. Mat. 15, 309-360 (1951) (Russian); Engl. transl. in Amer. Math. Soc. Transl. Ser. 2, 1, 253-304 (1955).

[4] D. Bollé, F. Gesztesy, H. Grosse, W. Schweiger, and B. Simon, Witten index, axial anomaly, and Krein's spectral shift function in supersymmetric quantum mechanics, J. Math. Phys. 28, 1512-1525 (1987).

[5] S. Clark and F. Gesztesy, Weyl-Titchmarsh M-function asymptotics, local uniqueness results, trace formulas, and Borg-type theorems for Dirac operators, Trans. Amer. Math. Soc. 354, 3475-3534 (2002).

[6] J. Eckhardt, F. Gesztesy, R. Nichols, A. Sakhnovich, and G. Teschl, Inverse spectral problems for Schrödinger-type operators with distributional matrix-valued potentials, Diff. Integral Eq. 28, 505-522 (2015).

[7] J. Eckhardt, F. Gesztesy, R. Nichols, and G. Teschl, Supersymmetry and Schrödinger-type operators with distributional matrix-valued potential, J. Spectral Theory 4, 715-768 (2014).

[8] B. Fritzsche, B. Kirstein, I. Ya. Roitberg, and A. L. Sakhnovich, Recovery of Dirac system from the rectangular Weyl-Titchmarsh matrix function, Inverse Problems 28, 015010 (2012).

[9] F. Gesztesy, Inverse spectral theory as influenced by Barry Simon, in Spectral Theory and Mathematical Physics: A Festschrift in Honor of Barry Simon's 60th Birthday. Ergodic Schrödinger Operators, Singular Spectrum, Orthogonal Polynomials, and Inverse Spectral Theory, F. Gesztesy, P. Deift, C. Galvez, P. Perry, and W. Schlag (eds.), Proceedings of Symposia in Pure Mathematics, Amer. Math. Soc., Vol. 76/2, Providence, RI, 2007, pp. $741-820$.

[10] F. Gesztesy, J. A. Goldstein, H. Holden, and G. Teschl, Abstract wave equations and associated Dirac operators, Ann. Mat. Pura Appl. 191, 631-676 (2012).

[11] F. Gesztesy and M. Pang, On (conditional) positive semidefiniteness in a matrix-valued context, Studia Math. 236, 143-192 (2017).

[12] F. Gesztesy and A. Sakhnovich, in preparation.

[13] F. Gesztesy, W. Schweiger, and B. Simon, Commutation methods applied to the mKdVequation, Trans. Amer. Math. Soc. 324, 465-525 (1991).

[14] F. Gesztesy and B. Simon, A new approach to inverse spectral theory, II. General real potentials and the connection to the spectral measure, Ann. Math. 152, 593-643 (2000).

[15] I. C. Gohberg and M. G. Krein, Theory and Applications of Volterra Operators in Hilbert Space, Transl. Math. Monogr., Vol. 24, Amer. Math. Soc., Providence, RI, 1970.

[16] M. Hitrik, Properties of the scattering transform on the real line, J. Math. Anal. Appl. 258, 223-243 (2001).

[17] B. M. Levitan, Inverse Sturm-Liouville Problems, VNU Science Press, Utrecht, 1987.

[18] B. M. Levitan and M. G. Gasymov, Determination of a differential equation by two of its spectra, Russian Math. Surv. 19:2, 1-63 (1964).

[19] B. M. Levitan and I. S. Sargsjan, Introduction to Spectral Theory, Transl. Math. Monographs 39, Amer. Math. Soc., Providence, RI, 1975.

[20] E. Lukacs, A survey of the theory of characteristic functions, Adv. Appl. Probability 4, 1-38 (1972).

[21] V. A. Marchenko, Sturm-Liouville Operators and Applications, revised ed., AMS, Chelsea, Providence, RI, 2011. 
[22] A. I. Markushevich, Theory of Analytic Functions of a Complex Variable, Three volumes in one, 2nd rev. ed., Chelsea, New York, 1985.

[23] C. Remling, Schrödinger operators and de Branges spaces, J. Funct. Anal. 196, 323-394 (2002).

[24] C. Remling, Inverse spectral theory for one-dimensional Schrödinger operators: The A function, Math. Z. 245, 597-617 (2003).

[25] A. L. Sakhnovich, Asymptotics of spectral functions of an S-colligation, Soviet Math. (Iz. VUZ) 32, 92-105 (1988).

[26] A. L. Sakhnovich, Dirac type and canonical systems: spectral and Weyl-Titchmarsh functions, direct and inverse problems, Inverse Problems 18, 331-348 (2002).

[27] A. L. Sakhnovich, Inverse problem for Dirac systems with locally square-summable potentials and rectangular Weyl-Titchmarsh functions, J. Spectral Theory 5, 547-569 (2015).

[28] A. L. Sakhnovich, L. A. Sakhnovich, and I. Ya. Roitberg, Inverse Problems and Nonlinear Evolution Equations. Solutions, Darboux Matrices and Weyl-Titchmarsh Functions, De Gruyter Studies in Mathematics, Vol. 47, De Gruyter, Berlin, 2013.

[29] L. A. Sakhnovich, Spectral analysis of Volterra's operators defined in the space of vectorfunctions $L^{2}$, Ukrain. Mat. Zh. 16, 259-268 (1964). English transl. Amer. Math. Soc. Transl. (2) 61, 85-95 (1967).

[30] Z. Sasvari, Multivariate Characteristic and Correlation Functions, De Gruyter Studies in Mathematics, Vol. 50, De Gruyter, Berlin, 2013.

[31] B. Simon, A new approach to inverse spectral theory, I. Fundamental formalism, Ann. Math. 150, 1029-1057 (1999).

[32] Y. Zhang, The One Dimensional Inverse Problem and New Integrable Dynamical Systems, Ph.D. Thesis, Univ. of Rochester, NY, 2003.

[33] Y. Zhang, Solvability of a class of integro-differential equations and connections to one dimensional inverse problems, J. Math. Anal. Appl. 321, 286-298 (2006).

Department of Mathematics, Baylor University, One Bear Place \#97328, Waco, TX 76798-7328, USA

E-mail address: Fritz_Gesztesy@baylor.edu

$U R L:$ http://www . baylor .edu/math/index.php?id=935340

Faculty of Mathematics, University of Vienna, Oskar-Morgenstern-Platz 1, 1090 Wien, Austria

E-mail address: oleksandr.sakhnovych@univie.ac.at

$U R L:$ http://www.mat.univie.ac.at/ sakhnov/ 